\title{
Fractional Directional Differentiation and Its Application for Multiscale Texture Enhancement
}

\author{
Chaobang Gao, ${ }^{1}$ Jiliu Zhou, $^{2}$ and Weihua Zhang ${ }^{2}$ \\ ${ }^{1}$ College of Information Science and Technology, Chengdu University, Chengdu 610106, China \\ ${ }^{2}$ School of Computer Science, Sichuan University, Chengdu 610064, China \\ Correspondence should be addressed to Chaobang Gao, kobren427@163.com
}

Received 11 June 2012; Revised 24 July 2012; Accepted 26 July 2012

Academic Editor: Kwok-Wo Wong

Copyright (c) 2012 Chaobang Gao et al. This is an open access article distributed under the Creative Commons Attribution License, which permits unrestricted use, distribution, and reproduction in any medium, provided the original work is properly cited.

This paper derives the directional derivative expression of Taylor formula for two-variable function from Taylor formula of one-variable function. Further, it proposes a new concept, fractional directional differentiation (FDD), and corresponding theories. To achieve the numerical calculation, the paper deduces power series expression of FDD. Moreover, the paper discusses the construction of FDD mask in the four quadrants, respectively, for digital image. The differential coefficients of every direction are not the same along the eight directions in the four quadrants, which is the biggest difference by contrast to general fractional differentiation and can reflect different fractional change rates along different directions, and this benefits to enlarge the differences among the image textures. Experiments show that, for texture-rich digital images, the capability of nonlinearly enhancing comprehensive texture details by FDD is better than those by the general fractional differentiation and Butterworth filter. By quantity analysis, it shows that state-of-the-art effect of texture enhancement is obtained by FDD.

\section{Introduction}

Fractional differentiation, also called noninteger differentiation, is not a new concept: it dates back to Cauchy, Riemann, Liouville, and Letnikov in the 19th century. Then, several theoretical physicists and mathematicians have studied fractional differential equations, especially fractional-order linear differential equations and fractional differential equations with delay [1-5], also fractional equations of fractional variational problems [6, 7]. In comparison with integer-order differentiation, the fractional differentiation of direct current or low frequency signal is often nonzero. Fractional differential processing is not only nonlinearly keeping signal's low-frequency and direct current components, but also nonlinearly enhancing the signal's high-frequency and middle-frequency components [8]. Therefore, special Mach's phenomenon appears in fractional differentiation of image. Moreover, it has a special bionic 
vision receptive field model for fractional differential antagonism characteristics $[9,10]$. Based on the special characteristics of fractional differentiation, in the last two decades, fractional differentiation has played a very important role in various physical sciences fields, such as mechanics, electricity, chemistry, biology, economics, time and frequency domains, system identification, notably control theory, mechatronics, and robotics $[9,11,12]$. Recently, fractal theory has already been used in fractal image processing [13-16].

In image processing, texture enhancement is an important issue in many areas like pattern recognition, image restoration, medical imaging processing, robotics, interpretation of image data, and remote sensing. But, to our knowledge, only a few reliable methods focus on texture enhancing. Texture enhancement often makes use of integer-order differential operators, especially 1-order used by the gradient and 2-order by the Laplacian. In [1316], the principles of fractional differential operators in image processing are introduced with implementation of fractional differential masks. But these fractional differential masks have a common flaw which the fractional differential coefficients of every direction are always the same along the eight directions in the image plane, which is not conducive to reflect the different change rates of the image along different directions and is not benefitial to enlarge the differences among textures. Based on this, we introduce the concept of directional derivative to fractional differentiation and propose a new mathematical method, fractional directional differentiation (FDD), and then try to employ it to image processing, particularly to comprehensive fractal-like texture-details enhancement. Furthermore, by taking the approximations of power series expression of FDD, we propose four novel FDD masks and corresponding numerical calculation rules. The fractional differential coefficients along the eight directions in the image plane are not the same, which can reflect different fractional change rates along different directions and is benefit to enlarge the differences among the image textures. Thus, this can lead to texture enhancement. Experiments show that FDD can effectively enhance texture details of image and has a greater calculation range than that of fractional differential for texture-enhancing.

This paper is organized as follows. Section 2 first recalls on the necessary theoretical background of directional derivative and the main definitions of Riemann-Liouville in Euclidean space. According to Taylor formula of one-variable function, we deduce the directional derivative expression of Taylor formula for two-variable function, give the definition of FDD, and further deduce power series expression of FDD. Section 3 deals with and gives four FDD masks and their calculation rules. Section 4 reports the nonlinearly enhancing capability of texture details based on FDD. By calculating five classical parameters from gray-level cooccurrence matrix in particular, information entropy, average gradient and projection of gray-level, we implement quantity analysis.

\section{Theory of FDD}

\subsection{Directional Derivative}

Assume that $z=f(x, y)$ is defined in the neighborhood $D$. Find the line which passes through $M_{0}\left(x_{0}, y_{0}\right)$ and parallels to $l$ as follows:

$$
\frac{x-x_{0}}{\cos \alpha}=\frac{y-y_{0}}{\sin \alpha}=t, \quad l=(\cos \alpha, \sin \alpha),
$$


where $\alpha$ is the direction angle. Let $M\left(x_{0}+\Delta x, y_{0}+\Delta y\right)$ satisfy $(2.1)$ and $h=\operatorname{sgn}(t) \cdot \overline{M_{0} M}$. If we take the direction of the vector $M_{0} M$ the same as $l$, then $t>0, h>0$, otherwise, $t<0, h<0$, that is to say, $h$ changes along the positive or negative direction of $l$. If the limit

$$
\lim _{h \rightarrow 0} \frac{f(M)-f\left(M_{0}\right)}{h}
$$

exists, then (2.2) is called the directional derivative of $f(x, y)$ along the direction $l$ at $M_{0}$, write $\partial f\left(M_{0}\right) / \partial l$, which denotes the slope of tangent line at $M_{0}$ for the plane curve

$$
\left\{\begin{array}{l}
z=f(x, y) \\
\frac{x-x_{0}}{\cos \alpha}=\frac{y-y_{0}}{\sin \alpha}
\end{array}\right.
$$

and the geometric meaning is the same as general derivative.

It is well known, the sufficient condition which (2.2) exists is that $f(x, y)$ has continuous partial derivatives of first order in the neighborhood $D$ at the point $M_{0}$ and

$$
\frac{\partial f\left(M_{0}\right)}{\partial l}=\frac{\partial f\left(M_{0}\right)}{\partial x} \cos \alpha+\frac{\partial f\left(M_{0}\right)}{\partial y} \sin \alpha .
$$

Clearly, for any $M_{0}\left(x_{0}, y_{0}\right)$ in $D, \partial f\left(M_{0}\right) / \partial l$ is the function with respect to $x$ and $y$, that is,

$$
\frac{\partial f(x, y)}{\partial l}=\frac{\partial f(x, y)}{\partial x} \cos \alpha+\frac{\partial f(x, y)}{\partial y} \sin \alpha .
$$

If $f(x, y)$ exists continuous partial derivatives of second order in the neighborhood $D$, making the direction $l$ fixed and by (2.5), we obtain

$$
\frac{\partial^{2} f}{\partial l^{2}}=\frac{\partial^{2} f}{\partial x^{2}} \cos ^{2} \alpha+2 \frac{\partial^{2} f}{\partial x \partial y} \sin \alpha \cos \alpha+\frac{\partial^{2} f}{\partial y^{2}} \sin ^{2} \alpha \triangleq\left(\cos \alpha \frac{\partial}{\partial x}+\sin \alpha \frac{\partial}{\partial y}\right)^{2} f(x, y)
$$

Thus (2.6) is called the second order directional derivative of $f(x, y)$ along the direction $l$ at $M$.

Similarly, if $f(x, y)$ exists $n+1$ order continuous partial derivatives in $D$, we can define the $k$-order directional derivative of $f(x, y)$ along the direction $l$ at $M$ as follows:

$$
\frac{\partial^{k} f}{\partial l^{k}}=\left(\cos \alpha \frac{\partial}{\partial x}+\sin \alpha \frac{\partial}{\partial y}\right)^{k} f(x, y), \quad k=1,2, \ldots, n, n+1
$$

\subsection{Directional Derivative Expression of Taylor Formula for Two-Variable Function}

In [17], the author deduced the Taylor series expansion of multivariable function and gave its conditions of convergence. On this basis, we will further derive the directional derivative 
expression of Taylor formula for multi-variable function. In this paper, we only discuss twovariable function. Actually, for multi-variable function, it is similar to two-variable function.

Let $f(x, y)$ have $n+1$ order continuous partial derivatives in $D$, then (2.7) holds and we call that

$$
d^{k} f=\left(\Delta x \frac{\partial}{\partial x}+\Delta y \frac{\partial}{\partial y}\right)^{k} f(x, y), \quad k=1, \ldots, n+1
$$

is the $k$-order $(k \in Z)$ differentiation of $f(x, y)$, thus the Taylor formula of $f(x, y)$ can be expressed as follows:

$$
f(x, y)=f\left(x_{0}, y_{0}\right)+d f+\frac{1}{2 !} d^{2} f+\cdots+\frac{1}{n !} d^{n} f+\frac{1}{(n+1) !} d^{n+1} f\left(x_{0}+\theta \Delta x, y_{0}+\theta \Delta y\right),
$$

where $0<\theta<1$. Now let $\cos \alpha=\Delta x / h, \sin \alpha=\Delta y / h, h=\operatorname{sgn}(t) \cdot \overline{M_{0} M}$, that is, when $t>0$, $h=\overline{M_{0} M}$, and when $t<0, h=-\overline{M_{0} M}$. Clearly, (2.8) becomes

$$
d^{k} f=h^{k}\left(\frac{\Delta x}{h} \frac{\partial}{\partial x}+\frac{\Delta y}{h} \frac{\partial}{\partial y}\right)^{k} f(x, y)=h^{k} \frac{\partial^{k} f}{\partial l^{k}}, \quad k=1,2, \ldots, n+1
$$

Taking (2.10) into (2.9), we get

$$
\begin{aligned}
f(x, y)= & f\left(x_{0}, y_{0}\right)+h \frac{\partial f}{\partial l}+\frac{h^{2}}{2 !} \frac{\partial^{2} f}{\partial l^{2}}+\cdots+\frac{h^{n}}{n !} \frac{\partial^{n} f}{\partial l^{n}} \\
& +\frac{h^{n+1}}{(n+1) !} \frac{\partial^{n+1} f\left(x_{0}+\theta \Delta x, y_{0}+\theta \Delta y\right)}{\partial l^{n+1}}, \quad 0<\theta<1 .
\end{aligned}
$$

Equation (2.11) is called the directional derivative expression of Taylor formula for $f(x, y)$. Compared with Taylor formula of one-variable function, they have a unified form.

\subsection{Fractional Differentiation}

If the signal $f(t)$ has $n+1$ order continuous derivatives and $n$ is at least $[v]=m-1(v \in$ $R, m \in Z$ ), where $[v]$ denotes the greatest integer and is less than or equal to $v$, then the definitions of fractional calculus based on Grumwald-letnikov and Riemann-Liouville [4] are equivalent. Otherwise, the latter is an expansion of the former with wider applications [10]. Though the definition of fractional calculus based on Riemann-Liouville is more common, few studies involve in its numerical solution. Currently, more researches still focus on the numerical solution of fractional calculus based on Grumwald-Letnikov [10, 13-15, 18]. It is 
well-known that $v$-order fractional integral based on Riemann-Liouville can be expressed as follows [1]:

$$
{ }_{0}^{R} I_{x}^{v} f(x)=\frac{1}{\Gamma(v)} \int_{0}^{x}(x-t)^{v-1} f(t) d t
$$

where $f(x)$ is continuous in $[0, x)$, and $v$ is a positive real number.

On the basis of fractional integral, $v$-order fractional differentiation based on RiemannLiouville, write ${ }_{0}^{R} D_{x}^{v}$, can be defined. In fact, the $v$-order differential operator ${ }_{0}^{R} D_{x}^{v}$ can be

viewed as ${ }_{0}^{R} I_{x}^{-v}$. Let $-v=-k+r$, where $k=[v]+1, r=[v]+1-v$. Obviously, $0<r<1$ and $k-1<v<k$. Thus, $v$-order differentiation of $f(x)$ is

$$
\begin{aligned}
{ }_{0}^{R} D_{x}^{v} f(x) & ={ }_{0}^{R} I_{x}^{-v} f(x)={ }_{0}^{R} I_{x}^{-k+r} f(x)={ }_{0}^{R} I_{x}^{-k} \cdot{ }_{0}^{R} I_{x}^{r} f(x) \\
& =\left(\frac{d}{d x}\right)^{k}\left[\frac{1}{\Gamma(r)} \int_{0}^{x}(x-t)^{r-1} f(t) d t\right] .
\end{aligned}
$$

Equation (2.13) shows obviously that the calculation of $v$-order fractional differentiation based on Riemann-Liouville need to first find $r$-order fractional integral, and then seek general $k$-order derivative. Note that ${ }_{0}^{R} I_{x}^{-k}$ and ${ }_{0}^{R} I_{x}^{r}$ must keep their order, which means that we must first find integral, then do derivative.

\subsection{FDD of Two-Variable Function}

In (2.11), when the direction $l$ is fixed at any point $M(x, y), h$ is a variable number along $l$. Thus, (2.11) can be seen as a function with respect to the variable $h$ and denotes the variety of $f(x, y)$ along the direction $l$ at $M(x, y)$. With the change of direction $l,(2.11)$ denotes the variety of $f(x, y)$ along different directions at $M(x, y)$. In order to quantify the variety of $f(x, y)$ in a certain direction $l$ at the point $M(x, y)$, find fractional differentiation (v-order) of (2.11) as follows:

$$
{ }_{0}^{R} D_{h}^{v} f(x, y)=\sum_{k=0}^{n} \frac{1}{k !} \frac{\partial^{k} f}{\partial l^{k}}{ }_{0}^{R} D_{h}^{v} h^{k}+\frac{1}{(n+1) !} \frac{\partial^{n+1} f\left(x_{0}+\theta \Delta x, y_{0}+\theta \Delta y\right)}{\partial l^{n+1}}{ }_{0}^{R} D_{h}^{v} h^{n+1},
$$

where $0<\theta<1$. Equation (2.14) denotes just the fractional measurement of the variety of $f(x, y)$ along the direction $l$ at $M_{0}\left(x_{0}, y_{0}\right)$. Equation (2.14) is called fractional directional differentiation (FDD) of $f(x, y)$ along the direction $l$ at $M_{0}\left(x_{0}, y_{0}\right)$. Thus, the FDD depicts a fractional change rate of $f(x, y)$ along the direction $l$ at any point $M(x, y)$.

\section{FDD Mask and Algorithm}

There are many studies on numerical calculation of fractional differentiation $[1,13,16,18]$. Similarly, we need to discuss numerical calculation of FDD. To implement numerical 
calculation of (2.14), we first seek its power series expression. According to [18], ${ }_{0}^{R} D_{h}^{v} h^{u}(u>$ $-1)$ can be induced as follows:

$$
\begin{aligned}
{ }_{0}^{R} D_{h}^{v}\left(h^{u}\right) & ={ }_{0}^{R} I_{h}^{-v}\left(h^{u}\right)={ }_{0}^{R} I_{h}^{-k}{ }_{0}^{R} I_{h}^{r}\left(h^{u}\right)=\left(\frac{d}{d h}\right)^{k}\left[\frac{1}{\Gamma(r)} \int_{0}^{h}(h-t)^{r-1} t^{u} d t\right] \\
& \stackrel{y=t / h}{=}\left(\frac{d}{d h}\right)^{k}\left[\frac{h^{u+r}}{\Gamma(r)} \int_{0}^{1}(1-y)^{r-1} y^{u} d y\right]=\left(\frac{d}{d h}\right)^{k}\left[\frac{h^{u+r}}{\Gamma(r)} B(r, u+1)\right] \\
& =\left(\frac{d}{d h}\right)^{k}\left[\frac{h^{u+r}}{\Gamma(r)} \frac{\Gamma(r) \Gamma(u+1)}{\Gamma(u+r+1)}\right]=\frac{\Gamma(u+1)}{\Gamma(u+r+1)}\left(\frac{d}{d h}\right)^{k} h^{u+r} \\
& =\frac{\Gamma(u+1)}{\Gamma(u+r+1)} \frac{\Gamma(u+r+1)}{\Gamma(u+r-k+1)} h^{u+r-k} \\
& =\frac{\Gamma(u+1)}{\Gamma(u-v+1)} h^{u-v},
\end{aligned}
$$

where $B(x, y)$ is the Beta function, and $n-1<v<n, n$ is an integer. Taking (3.1) into (2.14), we obtain

$$
{ }_{0}^{R} D_{h}^{v} f(x, y)=\sum_{n=0}^{\infty} \frac{1}{\Gamma(n-v+1)} \frac{\partial^{n} f}{\partial l^{n}} h^{n-v}
$$

Thus, (3.2) is the power series expression of FDD for $f(x, y)$.

Since the processing data of computer or digital filter is digital and finite, the maximum variety of image's grayscale is also finite. The shortest neighborhood distance between pixels can achieve only one pixel $[13,16]$. Therefore, the measurement for duration of two-dimensional digital image on $x$-coordinate or $y$-coordinate must take pixel as unit, and the minimum division must be $\Delta x=1, \Delta y=1$, and since $\cos \alpha=\Delta x / h$ or $\sin \alpha=\Delta y / h$, it has

$$
\Delta x=h \cos \alpha, \quad \Delta y=h \sin \alpha .
$$

Next, we will implement numerical calculation and seek the masks of FDD in four quadrants, respectively. In the first quadrant, the forward differences of $\partial f / \partial x, \partial f / \partial y$, $\partial^{2} f / \partial x^{2}, \partial^{2} f / \partial y^{2}$, and $\partial^{2} f / \partial x \partial y$ can be expressed as:

$$
\begin{gathered}
\frac{\partial f\left(x_{0}, y_{0}\right)}{\partial x} \approx f\left(x_{0}+1, y_{0}\right)-f\left(x_{0}, y_{0}\right), \\
\frac{\partial f\left(x_{0}, y_{0}\right)}{\partial y} \approx f\left(x_{0}, y_{0}+1\right)-f\left(x_{0}, y_{0}\right), \\
\frac{\partial^{2} f\left(x_{0}, y_{0}\right)}{\partial x^{2}} \approx f\left(x_{0}+2, y_{0}\right)-2 f\left(x_{0}+1, y_{0}\right)+f\left(x_{0}, y_{0}\right),
\end{gathered}
$$


Mathematical Problems in Engineering

$$
\begin{gathered}
\frac{\partial^{2} f\left(x_{0}, y_{0}\right)}{\partial y^{2}} \approx f\left(x_{0}, y_{0}+2\right)-2 f\left(x_{0}, y_{0}+1\right)+f\left(x_{0}, y_{0}\right) \\
\frac{\partial^{2} f\left(x_{0}, y_{0}\right)}{\partial x \partial y} \approx f\left(x_{0}+1, y_{0}+1\right)-f\left(x_{0}+1, y_{0}\right)-f\left(x_{0}, y_{0}+1\right)+f\left(x_{0}, y_{0}\right) .
\end{gathered}
$$

Taking (3.4) into (3.2) and by (2.7), (3.3), when $n=2$ in (3.2), we get

$$
\begin{aligned}
& { }_{0}^{R} D_{h}^{v} f\left(x_{0}, y_{0}\right) \\
& \approx \frac{h^{-v}}{\Gamma(1-v)} f\left(x_{0}, y_{0}\right)+\frac{h^{1-v}}{\Gamma(2-v)}\left(\frac{\partial f\left(x_{0}, y_{0}\right)}{\partial x} \cos \alpha+\frac{\partial f\left(x_{0}, y_{0}\right)}{\partial y} \sin \alpha\right) \\
& +\frac{h^{2-v}}{\Gamma(3-v)}\left(\frac{\partial^{2} f\left(x_{0}, y_{0}\right)}{\partial x^{2}} \cos ^{2} \alpha+2 \frac{\partial^{2} f\left(x_{0}, y_{0}\right)}{\partial x \partial y} \sin \alpha \cos \alpha+\frac{\partial^{2} f\left(x_{0}, y_{0}\right)}{\partial y^{2}} \sin ^{2} \alpha\right) \\
& =\frac{h^{-v}}{\Gamma(1-v)} f\left(x_{0}, y_{0}\right)+\frac{h^{1-v}}{\Gamma(2-v)} f\left(x_{0}+1, y_{0}\right) \cos \alpha-\frac{h^{1-v}}{\Gamma(2-v)} f\left(x_{0}, y_{0}\right) \cos \alpha \\
& +\frac{h^{1-v}}{\Gamma(2-v)} f\left(x_{0}, y_{0}+1\right) \sin \alpha-\frac{h^{1-v}}{\Gamma(2-v)} f\left(x_{0}, y_{0}\right) \sin \alpha \\
& +\frac{h^{2-v}}{\Gamma(3-v)} f\left(x_{0}+2, y_{0}\right) \cos ^{2} \alpha-2 \frac{h^{2-v}}{\Gamma(3-v)} f\left(x_{0}+1, y_{0}\right) \cos ^{2} \alpha \\
& +\frac{h^{2-v}}{\Gamma(3-v)} f\left(x_{0}, y_{0}\right) \cos ^{2} \alpha+2 \frac{h^{2-v}}{\Gamma(3-v)} f\left(x_{0}+1, y_{0}+1\right) \sin \alpha \cos \alpha \\
& -2 \frac{h^{2-v}}{\Gamma(3-v)} f\left(x_{0}+1, y_{0}\right) \sin \alpha \cos \alpha-2 \frac{h^{2-v}}{\Gamma(3-v)} f\left(x_{0}, y_{0}+1\right) \sin \alpha \cos \alpha \\
& +2 \frac{h^{2-v}}{\Gamma(3-v)} f\left(x_{0}, y_{0}\right) \sin \alpha \cos \alpha+\frac{h^{2-v}}{\Gamma(3-v)} f\left(x_{0}, y_{0}+2\right) \sin ^{2} \alpha \\
& -2 \frac{h^{2-v}}{\Gamma(3-v)} f\left(x_{0}, y_{0}+1\right) \sin ^{2} \alpha+\frac{h^{2-v}}{\Gamma(3-v)} f\left(x_{0}, y_{0}\right) \sin ^{2} \alpha \\
& =\left[\frac{h^{-v}}{\Gamma(1-v)}-\frac{h^{1-v} \cos \alpha+h^{1-v} \sin \alpha}{\Gamma(2-v)}+\frac{h^{2-v} \cos ^{2} \alpha+2 h^{2-v} \sin \alpha \cos \alpha+h^{2-v} \sin ^{2} \alpha}{\Gamma(3-v)}\right] \\
& \times f\left(x_{0}, y_{0}\right)+\left[\frac{h^{1-v} \cos \alpha}{\Gamma(2-v)}-\frac{2 h^{2-v} \cos ^{2} \alpha+2 h^{2-v} \sin \alpha \cos \alpha}{\Gamma(3-v)}\right] f\left(x_{0}+1, y_{0}\right) \\
& +\left[\frac{h^{2-v} \cos ^{2} \alpha}{\Gamma(3-v)}\right] f\left(x_{0}+2, y_{0}\right)+\left[\frac{h^{1-v} \sin \alpha}{\Gamma(2-v)}-\frac{2 h^{2-v} \sin \alpha \cos \alpha+2 h^{2-v} \sin ^{2} \alpha}{\Gamma(3-v)}\right] \\
& \times f\left(x_{0}, y_{0}+1\right)+\left[\frac{2 h^{2-v} \sin \alpha \cos \alpha}{\Gamma(3-v)}\right] f\left(x_{0}+1, y_{0}+1\right)+\left[\frac{h^{2-v} \sin ^{2} \alpha}{\Gamma(3-v)}\right] f\left(x_{0}, y_{0}+2\right) \text {. }
\end{aligned}
$$




\begin{tabular}{|c|c|c|}
\hline$a_{10}$ & $a_{13}$ & $a_{15}$ \\
\hline$a_{11}$ & $a_{14}$ & 0 \\
\hline$a_{12}$ & 0 & 0 \\
\hline
\end{tabular}

Figure 1: Mask FDD-1.

Let

$$
\begin{aligned}
& a_{10}=\frac{h^{-v}}{\Gamma(1-v)}-\frac{h^{1-v} \cos \alpha+h^{1-v} \sin \alpha}{\Gamma(2-v)}+\frac{h^{2-v} \cos ^{2} \alpha+2 h^{2-v} \sin \alpha \cos \alpha+h^{2-v} \sin ^{2} \alpha}{\Gamma(3-v)}, \\
& a_{11}=\frac{h^{1-v} \cos \alpha}{\Gamma(2-v)}-\frac{2 h^{2-v} \cos ^{2} \alpha+2 h^{2-v} \sin \alpha \cos \alpha}{\Gamma(3-v)}, \\
& a_{12}=\frac{h^{2-v} \cos ^{2} \alpha}{\Gamma(3-v)} \\
& a_{13}=\frac{h^{1-v} \sin \alpha}{\Gamma(2-v)}-\frac{2 h^{2-v} \sin \alpha \cos \alpha+2 h^{2-v} \sin ^{2} \alpha}{\Gamma(3-v)}, \\
& a_{14}=\frac{2 h^{2-v} \sin \alpha \cos \alpha}{\Gamma(3-v)}, \\
& a_{15}=\frac{h^{2-v} \sin ^{2} \alpha}{\Gamma(3-v)} .
\end{aligned}
$$

Then, (3.5) becomes

$$
\begin{aligned}
{ }_{0}^{R} D_{h}^{v} f\left(x_{0}, y_{0}\right) \approx & a_{10} f\left(x_{0}, y_{0}\right)+a_{11} f\left(x_{0}+1, y_{0}\right)+a_{12} f\left(x_{0}+2, y_{0}\right) \\
& +a_{13} f\left(x_{0}, y_{0}+1\right)+a_{14} f\left(x_{0}+1, y_{0}+1\right)+a_{15} f\left(x_{0}, y_{0}+2\right)
\end{aligned}
$$

Equation (3.7) indicates that it has a $3 \times 3$ mask when $n=2$, write FDD-1, shown in Figure 1 . In the first quadrant, we choose three direction angles $\alpha=0, \pi / 4$, and $\pi / 2$ to calculate the coefficients (3.6) of the mask FDD-1.

(i) For $\alpha=0$, let $h$ change strictly along the positive direction of $l=(1,0)$ in the image plane, therefore $h>0$ and we can find the FDD at $h=1$. By (3.3), we have $h \cos \alpha=$ $1, h \sin \alpha=0$. Thus, the coefficients (3.6) of the mask FDD- 1 becomes

$$
\begin{gathered}
a_{10}=\frac{1}{\Gamma(1-v)}-\frac{1}{\Gamma(2-v)}+\frac{1}{\Gamma(3-v)}, \quad a_{11}=\frac{1}{\Gamma(2-v)}-\frac{2}{\Gamma(3-v)} \\
a_{12}=\frac{1}{\Gamma(3-v)}, \quad a_{13}=0, \quad a_{14}=0, \quad a_{15}=0 .
\end{gathered}
$$


(ii) For $\alpha=\pi / 4$, let $h$ change always along the positive direction of $l=(\sqrt{2} / 2, \sqrt{2} / 2)$ in the image plane, therefore $h>0$ and FDD can be found at $h=\sqrt{2}$. By (3.3), we have $h \cos \alpha=1, h \sin \alpha=1$. Thus the coefficients (3.6) of the mask FDD- 1 are as follows:

$$
\begin{gathered}
a_{10}=\frac{\sqrt{2}^{-v}}{\Gamma(1-v)}-\frac{2 \sqrt{2}^{-v}}{\Gamma(2-v)}+\frac{4 \sqrt{2}^{-v}}{\Gamma(3-v)^{-v}}, \quad a_{11}=\frac{\sqrt{2}^{-v}}{\Gamma(2-v)}-\frac{4 \sqrt{2}^{-v}}{\Gamma(3-v)^{\prime}} \\
a_{12}=\frac{\sqrt{2}^{-v}}{\Gamma(3-v)}, \quad a_{13}=\frac{\sqrt{2}^{-v}}{\Gamma(2-v)}-\frac{4 \sqrt{2}^{-v}}{\Gamma(3-v)}, \quad a_{14}=\frac{2 \sqrt{2}^{-v}}{\Gamma(3-v)} \\
a_{15}=\frac{\sqrt{2}^{-v}}{\Gamma(3-v)} .
\end{gathered}
$$

(iii) For $\alpha=\pi / 2$, let $h$ change always along the positive direction of $l=(0,1)$ in the image plane, therefore $h>0$ and we can find the FDD at $h=1$. By (3.3), we have $h \cos \alpha=0, h \sin \alpha=1$. Thus the coefficients (3.6) of the mask FDD- 1 are equal to

$$
\begin{gathered}
a_{10}=\frac{1}{\Gamma(1-v)}-\frac{1}{\Gamma(2-v)}+\frac{1}{\Gamma(3-v)}, \quad a_{11}=0, \quad a_{12}=0 \\
a_{13}=\frac{1}{\Gamma(2-v)}-\frac{2}{\Gamma(3-v)}, \quad a_{14}=0, \quad a_{15}=\frac{1}{\Gamma(3-v)} .
\end{gathered}
$$

Similarly, in the second quadrant, the backward difference on the negative $x$ coordinate and forward difference on the positive $y$-coordinate of $\partial f / \partial x, \partial f / \partial y, \partial^{2} f / \partial x^{2}$, $\partial^{2} f / \partial y^{2}$, and $\partial^{2} f / \partial x \partial y$ are expressed as follows:

$$
\begin{gathered}
\frac{\partial f\left(x_{0}, y_{0}\right)}{\partial x} \approx f\left(x_{0}, y_{0}\right)-f\left(x_{0}-1, y_{0}\right), \\
\frac{\partial f\left(x_{0}, y_{0}\right)}{\partial y} \approx f\left(x_{0}, y_{0}+1\right)-f\left(x_{0}, y_{0}\right), \\
\frac{\partial^{2} f\left(x_{0}, y_{0}\right)}{\partial x^{2}} \approx f\left(x_{0}, y_{0}\right)-2 f\left(x_{0}-1, y_{0}\right)+f\left(x_{0}-2, y_{0}\right), \\
\frac{\partial^{2} f\left(x_{0}, y_{0}\right)}{\partial x \partial y} \approx f\left(x_{0}, y_{0}+1\right)-f\left(x_{0}-1, y_{0}+1\right)-f\left(x_{0}, y_{0}\right)+f\left(x_{0}-1, y_{0}\right), \\
\frac{\partial^{2} f\left(x_{0}, y_{0}\right)}{\partial y^{2}} \approx f\left(x_{0}, y_{0}+2\right)-2 f\left(x_{0}, y_{0}+1\right)+f\left(x_{0}, y_{0}\right) .
\end{gathered}
$$

Taking (3.11) into (3.2) and by (2.7), (3.3), where $n=2$ in (3.2), after simplification, we get

$$
\begin{aligned}
{ }_{0}^{R} D_{h}^{v} f\left(x_{0}, y_{0}\right) \approx & \frac{h^{-v}}{\Gamma(1-v)} f\left(x_{0}, y_{0}\right)+\frac{h^{1-v}}{\Gamma(2-v)}\left(\frac{\partial f\left(x_{0}, y_{0}\right)}{\partial x} \cos \alpha+\frac{\partial f\left(x_{0}, y_{0}\right)}{\partial y} \sin \alpha\right) \\
& +\frac{h^{2-v}}{\Gamma(3-v)}\left(\frac{\partial^{2} f}{\partial x^{2}} \cos ^{2} \alpha+2 \frac{\partial^{2} f}{\partial x \partial y} \sin \alpha \cos \alpha+\frac{\partial^{2} f}{\partial y^{2}} \sin ^{2} \alpha\right)
\end{aligned}
$$




$$
\begin{aligned}
= & \left(\frac{h^{-v}}{\Gamma(1-v)}+\frac{h^{1-v} \cos \alpha-h^{1-v} \sin \alpha}{\Gamma(2-v)}+\frac{h^{2-v}\left(\cos ^{2} \alpha-2 \sin \alpha \cos \alpha+\sin ^{2} \alpha\right)}{\Gamma(3-v)}\right) \\
& \times f\left(x_{0}, y_{0}\right)+\left(-\frac{h^{1-v} \cos \alpha}{\Gamma(2-v)}-\frac{2 h^{2-v} \cos ^{2} \alpha-2 h^{2-v} \sin \alpha \cos \alpha}{\Gamma(3-v)}\right) f\left(x_{0}-1, y_{0}\right) \\
& +\frac{h^{2-v} \cos ^{2} \alpha}{\Gamma(3-v)} f\left(x_{0}-2, y_{0}\right) \\
& +\left(\frac{h^{1-v} \sin \alpha}{\Gamma(2-v)}+\frac{2 h^{2-v} \sin \alpha \cos \alpha-2 h^{2-v} \sin ^{2} \alpha}{\Gamma(3-v)}\right) f\left(x_{0}, y_{0}+1\right) \\
& -\frac{2 h^{2-v} \sin \alpha \cos \alpha}{\Gamma(3-v)} f\left(x_{0}-1, y_{0}+1\right)+\frac{h^{2-v} \sin ^{2} \alpha}{\Gamma(3-v)} f\left(x_{0}, y_{0}+2\right) .
\end{aligned}
$$

Let

$$
\begin{aligned}
& a_{20}=\frac{h^{-v}}{\Gamma(1-v)}+\frac{h^{1-v}(\cos \alpha-\sin \alpha)}{\Gamma(2-v)}+\frac{h^{2-v}(\cos \alpha-\sin \alpha)^{2}}{\Gamma(3-v)}, \\
& a_{21}=-\frac{h^{1-v} \cos \alpha}{\Gamma(2-v)}-\frac{2 h^{2-v}\left(\cos ^{2} \alpha-\sin \alpha \cos \alpha\right)}{\Gamma(3-v)}, \\
& a_{22}=\frac{h^{2-v} \cos ^{2} \alpha}{\Gamma(3-v)}, \\
& a_{23}=\frac{h^{1-v} \sin \alpha}{\Gamma(2-v)}+\frac{2 h^{2-v}\left(\sin \alpha \cos \alpha-\sin ^{2} \alpha\right)}{\Gamma(3-v)}, \\
& a_{24}=-\frac{2 h^{2-v} \sin \alpha \cos \alpha}{\Gamma(3-v)}, \\
& a_{25}=\frac{h^{2-v} \sin ^{2} \alpha}{\Gamma(3-v)} .
\end{aligned}
$$

Then (3.12) can be expressed as

$$
\begin{aligned}
{ }_{0}^{R} D_{h}^{v} f\left(x_{0}, y_{0}\right) \approx & a_{20} f\left(x_{0}, y_{0}\right)+a_{21} f\left(x_{0}-1, y_{0}\right)+a_{22} f\left(x_{0}-2, y_{0}\right) \\
& +a_{23} f\left(x_{0}, y_{0}+1\right)+a_{24} f\left(x_{0}-1, y_{0}+1\right)+a_{25} f\left(x_{0}, y_{0}+2\right) .
\end{aligned}
$$

Equation (3.14) indicates that it has a $3 \times 3$ mask when $n=2$, write FDD-2, shown in Figure 2.

Since the mask FDD-2 in Figure 2 denotes the algorithm of FDD along different directions in the second quadrant, we can also take three direction angles $\alpha=0, \pi / 4$, and $\pi / 2$ to calculate the coefficients (3.13). In fact, the three direction angles in the second quadrant can show the direction angles $\alpha=\pi / 2,3 \pi / 4$, and $\pi$ in the image plane. Similar to the calculation of the mask FDD-1's coefficients, the calculation of (3.13) is as follows: 


\begin{tabular}{|l|c|c|}
\hline$a_{22}$ & 0 & 0 \\
\hline$a_{21}$ & $a_{24}$ & 0 \\
\hline$a_{20}$ & $a_{23}$ & $a_{25}$ \\
\hline
\end{tabular}

Figure 2: Mask FDD-2.

(i) When $\alpha=0$, we have

$$
\begin{gathered}
a_{20}=\frac{1}{\Gamma(1-v)}+\frac{1}{\Gamma(2-v)}+\frac{1}{\Gamma(3-v)}, \quad a_{21}=-\frac{1}{\Gamma(2-v)}-\frac{2}{\Gamma(3-v)}, \\
a_{22}=\frac{1}{\Gamma(3-v)}, \quad a_{23}=0, \quad a_{24}=0, \quad a_{25}=0 .
\end{gathered}
$$

(ii) When $\alpha=\pi / 4$ and by (3.3), we obtain

$$
\begin{array}{lll}
a_{20}=\frac{\sqrt{2}^{-v}}{\Gamma(1-v)}, & a_{21}=-\frac{\sqrt{2}^{-v}}{\Gamma(2-v)}, & a_{22}=\frac{\sqrt{2}^{-v}}{\Gamma(3-v)}, \\
a_{23}=\frac{\sqrt{2}^{-v}}{\Gamma(2-v)}, & a_{24}=-\frac{2 \sqrt{2}^{-v}}{\Gamma(3-v)}, & a_{25}=\frac{\sqrt{2}^{-v}}{\Gamma(3-v)} .
\end{array}
$$

(iii) When $\alpha=\pi / 2$ and by (3.3), it has

$$
\begin{gathered}
a_{20}=\frac{1}{\Gamma(1-v)}-\frac{1}{\Gamma(2-v)}+\frac{1}{\Gamma(3-v)}, \quad a_{21}=0, \quad a_{22}=0, \\
a_{23}=\frac{1}{\Gamma(2-v)}-\frac{2}{\Gamma(3-v)}, \quad a_{24}=0, \quad a_{25}=\frac{1}{\Gamma(3-v)} .
\end{gathered}
$$

In the same way, the backward differences of $\partial f / \partial x, \partial f / \partial y, \partial^{2} f / \partial x^{2}, \partial^{2} f / \partial y^{2}$, and $\partial^{2} f / \partial x \partial y$ in the third quadrant can be expressed as follows:

$$
\begin{gathered}
\frac{\partial f\left(x_{0}, y_{0}\right)}{\partial x} \approx f\left(x_{0}, y_{0}\right)-f\left(x_{0}-1, y_{0}\right), \\
\frac{\partial f\left(x_{0}, y_{0}\right)}{\partial y} \approx f\left(x_{0}, y_{0}\right)-f\left(x_{0}, y_{0}-1\right), \\
\frac{\partial^{2} f\left(x_{0}, y_{0}\right)}{\partial x^{2}} \approx f\left(x_{0}, y_{0}\right)-2 f\left(x_{0}-1, y_{0}\right)+f\left(x_{0}-2, y_{0}\right),
\end{gathered}
$$




$$
\begin{gathered}
\frac{\partial^{2} f\left(x_{0}, y_{0}\right)}{\partial x \partial y} \approx f\left(x_{0}, y_{0}\right)-f\left(x_{0}-1, y_{0}\right)-f\left(x_{0}, y_{0}-1\right)+f\left(x_{0}-1, y_{0}-1\right), \\
\frac{\partial^{2} f\left(x_{0}, y_{0}\right)}{\partial y^{2}} \approx f\left(x_{0}, y_{0}\right)-2 f\left(x_{0}, y_{0}-1\right)+f\left(x_{0}, y_{0}-2\right) .
\end{gathered}
$$

Taking (3.18) into (3.2) and by (2.7), (3.3), when $n=2$ in (3.2), after simplification, we get its approximation

$$
\begin{aligned}
{ }_{0}^{R} D_{h}^{v} f\left(x_{0}, y_{0}\right) \approx & \frac{h^{-v}}{\Gamma(1-v)} f\left(x_{0}, y_{0}\right)+\frac{h^{1-v}}{\Gamma(2-v)}\left(\frac{\partial f\left(x_{0}, y_{0}\right)}{\partial x} \cos \alpha+\frac{\partial f\left(x_{0}, y_{0}\right)}{\partial y} \sin \alpha\right) \\
& +\frac{h^{2-v}}{\Gamma(3-v)}\left(\frac{\partial^{2} f}{\partial x^{2}} \cos ^{2} \alpha+2 \frac{\partial^{2} f}{\partial x \partial y} \sin \alpha \cos \alpha+\frac{\partial^{2} f}{\partial y^{2}} \sin ^{2} \alpha\right) \\
= & \left(\frac{h^{-v}}{\Gamma(1-v)}+\frac{h^{1-v}(\cos \alpha+\sin \alpha)}{\Gamma(2-v)}+\frac{h^{2-v}(\cos \alpha+\sin \alpha)^{2}}{\Gamma(3-v)}\right) f\left(x_{0}, y_{0}\right) \\
& +\left(-\frac{h^{1-v} \cos \alpha}{\Gamma(2-v)}-\frac{2 h^{2-v}\left(\cos ^{2} \alpha+\sin \alpha \cos \alpha\right)}{\Gamma(3-v)}\right) f\left(x_{0}-1, y_{0}\right) \\
& +\frac{h^{2-v} \cos ^{2} \alpha}{\Gamma(3-v)} f\left(x_{0}-2, y_{0}\right) \\
& +\left(-\frac{h^{1-v} \sin \alpha}{\Gamma(2-v)}-\frac{2 h^{2-v}\left(\sin \alpha \cos \alpha+\sin ^{2} \alpha\right)}{\Gamma(3-v)}\right) f\left(x_{0}, y_{0}-1\right) \\
& +\frac{2 h^{2-v} \sin \alpha \cos \alpha}{\Gamma(3-v)} f\left(x_{0}-1, y_{0}-1\right) \\
& +\frac{h^{2-v} \sin ^{2} \alpha}{\Gamma(3-v)} f\left(x_{0}, y_{0}-2\right) .
\end{aligned}
$$

Let

$$
\begin{aligned}
& a_{30}=\frac{h^{-v}}{\Gamma(1-v)}+\frac{h^{1-v}(\cos \alpha+\sin \alpha)}{\Gamma(2-v)}+\frac{h^{2-v}(\cos \alpha+\sin \alpha)^{2}}{\Gamma(3-v)}, \\
& a_{31}=-\frac{h^{1-v} \cos \alpha}{\Gamma(2-v)}-\frac{2 h^{2-v}\left(\cos ^{2} \alpha+\sin \alpha \cos \alpha\right)}{\Gamma(3-v)}, \\
& a_{32}=\frac{h^{2-v} \cos ^{2} \alpha}{\Gamma(3-v)}, \\
& a_{33}=-\frac{h^{1-v} \sin \alpha}{\Gamma(2-v)}-\frac{2 h^{2-v}\left(\sin \alpha \cos \alpha+\sin ^{2} \alpha\right)}{\Gamma(3-v)},
\end{aligned}
$$




\begin{tabular}{|c|c|c|}
\hline 0 & 0 & $a_{32}$ \\
\hline 0 & $a_{34}$ & $a_{31}$ \\
\hline$a_{35}$ & $a_{33}$ & $a_{30}$ \\
\hline
\end{tabular}

Figure 3: Mask FDD-3.

$$
\begin{aligned}
& a_{34}=\frac{2 h^{2-v} \sin \alpha \cos \alpha}{\Gamma(3-v)}, \\
& a_{35}=\frac{h^{2-v} \sin ^{2} \alpha}{\Gamma(3-v)} .
\end{aligned}
$$

Thus, (3.19) can be rewritten as

$$
\begin{aligned}
{ }_{0}^{R} D_{h}^{v} f\left(x_{0}, y_{0}\right) \approx & a_{30} f\left(x_{0}, y_{0}\right)+a_{31} f\left(x_{0}-1, y_{0}\right)+a_{32} f\left(x_{0}-2, y_{0}\right) \\
& +a_{33} f\left(x_{0}, y_{0}-1\right)+a_{34} f\left(x_{0}-1, y_{0}-1\right)+a_{35} f\left(x_{0}, y_{0}-2\right) .
\end{aligned}
$$

Equation (3.21) indicates that it has a $3 \times 3$ mask when $n=2$, write FDD-3, shown in Figure 3 .

The same as the first and the second quadrants, because the mask in Figure 3 denotes the algorithm of FDD along different directions in the third quadrant, we can also take threedirections angles $\alpha=0, \pi / 4$, and $\pi / 2$ into account for (3.20). Actually, the three-direction angles in the third quadrant can denote the $\alpha=\pi, 5 \pi / 4$, and $3 \pi / 2$ in the image plane.

(i) For $\alpha=0$, we have

$$
\begin{gathered}
a_{30}=\frac{1}{\Gamma(1-v)}+\frac{1}{\Gamma(2-v)}+\frac{1}{\Gamma(3-v)}, \quad a_{31}=-\frac{1}{\Gamma(2-v)}-\frac{2}{\Gamma(3-v)}, \\
a_{32}=\frac{1}{\Gamma(3-v)}, \quad a_{33}=0, \quad a_{34}=0, \quad a_{35}=0 .
\end{gathered}
$$

(ii) For $\alpha=\pi / 4$ and by (3.3), we obtain

$$
\begin{array}{cc}
a_{30}=\frac{\sqrt{2}^{-v}}{\Gamma(1-v)}+\frac{2 \sqrt{2}^{-v}}{\Gamma(2-v)}+\frac{4 \sqrt{2}^{2-v}}{\Gamma(3-v)^{-v}}, \quad a_{31}=-\frac{\sqrt{2}^{-v}}{\Gamma(2-v)}-\frac{4 \sqrt{2}^{-v}}{\Gamma(3-v)^{\prime}}, \quad a_{32}=\frac{\sqrt{2}^{-v}}{\Gamma(3-v)^{\prime}}, \\
a_{33}=-\frac{\sqrt{2}^{-v}}{\Gamma(2-v)}-\frac{4 \sqrt{2}^{-v}}{\Gamma(3-v)}, \quad a_{34}=\frac{2 \sqrt{2}^{-v}}{\Gamma(3-v)}, \quad a_{35}=\frac{\sqrt{2}^{-v}}{\Gamma(3-v)} .
\end{array}
$$


(iii) For $\alpha=\pi / 2$, one has

$$
\begin{gathered}
a_{30}=\frac{h^{-v}}{\Gamma(1-v)}+\frac{h^{-v}}{\Gamma(2-v)}+\frac{h^{-v}}{\Gamma(3-v)}, \quad a_{31}=0, \\
a_{32}=0, \quad a_{33}=-\frac{h^{-v}}{\Gamma(2-v)}-\frac{2 h^{-v}}{\Gamma(3-v)}, \quad a_{34}=0, \quad a_{35}=\frac{h^{-v}}{\Gamma(3-v)} .
\end{gathered}
$$

Finally, in the fourth quadrant, the forward difference on positive $x$-coordinate and backward difference on negative $y$-coordinate of $\partial f / \partial x, \partial f / \partial y, \partial^{2} f / \partial x^{2}, \partial^{2} f / \partial y^{2}$, and $\partial^{2} f / \partial x \partial y$ are

$$
\begin{gathered}
\frac{\partial f\left(x_{0}, y_{0}\right)}{\partial x} \approx f\left(x_{0}+1, y_{0}\right)-f\left(x_{0}, y_{0}\right), \\
\frac{\partial f\left(x_{0}, y_{0}\right)}{\partial y} \approx f\left(x_{0}, y_{0}\right)-f\left(x_{0}, y_{0}-1\right), \\
\frac{\partial^{2} f\left(x_{0}, y_{0}\right)}{\partial x^{2}} \approx f\left(x_{0}+2, y_{0}\right)-2 f\left(x_{0}+1, y_{0}\right)+f\left(x_{0}, y_{0}\right), \\
\frac{\partial^{2} f\left(x_{0}, y_{0}\right)}{\partial y^{2}} \approx f\left(x_{0}, y_{0}\right)-2 f\left(x_{0}, y_{0}-1\right)+f\left(x_{0}, y_{0}-2\right) . \\
\frac{\partial^{2} f\left(x_{0}, y_{0}\right)}{\partial x \partial y} \approx f\left(x_{0}+1, y_{0}\right)-f\left(x_{0}, y_{0}\right)-f\left(x_{0}+1, y_{0}-1\right)+f\left(x_{0}, y_{0}-1\right) .
\end{gathered}
$$

Taking (3.25) into (3.2) and by (2.7), (3.3), when $n=2$ in (3.2), we obtain

$$
\begin{aligned}
{ }_{0}^{R} D_{h}^{v} f\left(x_{0}, y_{0}\right) \approx & \frac{h^{-v}}{\Gamma(1-v)} f\left(x_{0}, y_{0}\right)+\frac{h^{1-v}}{\Gamma(2-v)}\left(\frac{\partial f\left(x_{0}, y_{0}\right)}{\partial x} \cos \alpha+\frac{\partial f\left(x_{0}, y_{0}\right)}{\partial y} \sin \alpha\right) \\
& +\frac{h^{2-v}}{\Gamma(3-v)}\left(\frac{\partial^{2} f}{\partial x^{2}} \cos ^{2} \alpha+2 \frac{\partial^{2} f}{\partial x \partial y} \sin \alpha \cos \alpha+\frac{\partial^{2} f}{\partial y^{2}} \sin ^{2} \alpha\right) \\
= & \left(\frac{h^{-v}}{\Gamma(1-v)}-\frac{h^{1-v}(\cos \alpha-\sin \alpha)}{\Gamma(2-v)}+\frac{h^{2-v}(\cos \alpha-\sin \alpha)^{2}}{\Gamma(3-v)}\right) f\left(x_{0}, y_{0}\right) \\
& +\left(\frac{h^{1-v} \cos \alpha}{\Gamma(2-v)}+\frac{2 h^{2-v}\left(\sin \alpha \cos \alpha-\cos ^{2} \alpha\right)}{\Gamma(3-v)}\right) f\left(x_{0}+1, y_{0}\right) \\
& +\frac{h^{2-v} \cos ^{2} \alpha}{\Gamma(3-v)} f\left(x_{0}+2, y_{0}\right)
\end{aligned}
$$


Mathematical Problems in Engineering

$$
\begin{aligned}
& +\left(-\frac{h^{1-v} \sin \alpha}{\Gamma(2-v)}-\frac{2 h^{2-v}\left(\sin ^{2} \alpha-\sin \alpha \cos \alpha\right)}{\Gamma(3-v)}\right) f\left(x_{0}, y_{0}-1\right) \\
& +\left(-\frac{2 h^{2-v} \sin \alpha \cos \alpha}{\Gamma(3-v)}\right) f\left(x_{0}+1, y_{0}-1\right) \\
& +\frac{h^{2-v} \sin ^{2} \alpha}{\Gamma(3-v)} f\left(x_{0}, y_{0}-2\right) .
\end{aligned}
$$

Let

$$
\begin{aligned}
& a_{40}=\frac{h^{-v}}{\Gamma(1-v)}-\frac{h^{1-v}(\cos \alpha-\sin \alpha)}{\Gamma(2-v)}+\frac{h^{2-v}(\cos \alpha-\sin \alpha)^{2}}{\Gamma(3-v)}, \\
& a_{41}=\frac{h^{1-v} \cos \alpha}{\Gamma(2-v)}+\frac{2 h^{2-v}\left(\sin \alpha \cos \alpha-\cos ^{2} \alpha\right)}{\Gamma(3-v)}, \\
& a_{42}=\frac{h^{2-v} \cos ^{2} \alpha}{\Gamma(3-v)}, \\
& a_{43}=-\frac{h^{1-v} \sin \alpha}{\Gamma(2-v)}-\frac{2 h^{2-v}\left(\sin ^{2} \alpha-\sin \alpha \cos \alpha\right)}{\Gamma(3-v)}, \\
& a_{44}=-\frac{2 h^{2-v} \sin \alpha \cos \alpha}{\Gamma(3-v)}, \\
& a_{45}=\frac{h^{2-v} \sin ^{2} \alpha}{\Gamma(3-v)} .
\end{aligned}
$$

Then (3.26) becomes

$$
\begin{aligned}
{ }_{0}^{R} D_{h}^{v} f\left(x_{0}, y_{0}\right) \approx & a_{40} f\left(x_{0}, y_{0}\right)+a_{41} f\left(x_{0}+1, y_{0}\right)+a_{42} f\left(x_{0}, y_{0}-1\right) \\
& +a_{43} f\left(x_{0}+2, y_{0}\right)+a_{44} f\left(x_{0}+1, y_{0}-1\right)+a_{45} f\left(x_{0}, y_{0}-2\right)
\end{aligned}
$$

Equation (3.28) denotes that it has a $3 \times 3$ mask when $n=2$, write FDD-4, shown in Figure 4 .

Since the mask FDD- 4 in Figure 4 denotes the algorithm of FDD along different directions in fourth quadrant, we can also take the three direction angles $\alpha=0, \pi / 4$, and $\pi / 2$ into account for (3.27). Similarly, the three direction angles in the fourth quadrant can denote the $\alpha=3 \pi / 2,7 \pi / 4$, and $2 \pi$ in the image plane. 


\begin{tabular}{|c|c|c|}
\hline$a_{45}$ & $a_{43}$ & $a_{40}$ \\
\hline 0 & $a_{44}$ & $a_{41}$ \\
\hline 0 & 0 & $a_{42}$ \\
\hline
\end{tabular}

Figure 4: Mask FDD-4.

(i) When $\alpha=0$, it has

$$
\begin{gathered}
a_{40}=\frac{1}{\Gamma(1-v)}-\frac{1}{\Gamma(2-v)}+\frac{1}{\Gamma(3-v)}, \quad a_{41}=\frac{1}{\Gamma(2-v)}-\frac{2}{\Gamma(3-v)}, \\
a_{42}=\frac{1}{\Gamma(3-v)}, \quad a_{43}=0, \quad a_{44}=0, \quad a_{45}=0 .
\end{gathered}
$$

(ii) When $\alpha=\pi / 4$ and by (3.3), we have

$$
\begin{aligned}
a_{40}=\frac{\sqrt{2}^{-v}}{\Gamma(1-v)}, & a_{41}=\frac{\sqrt{2}^{-v}}{\Gamma(2-v)}, & a_{42}=\frac{\sqrt{2}^{-v}}{\Gamma(3-v)}, \\
a_{43}=-\frac{\sqrt{2}^{-v}}{\Gamma(2-v)}, & a_{44}=-\frac{2 \sqrt{2}^{-v}}{\Gamma(3-v)}, & a_{45}=\frac{\sqrt{2}^{-v}}{\Gamma(3-v)} .
\end{aligned}
$$

(iii) When $\alpha=\pi / 2$ and by (3.3), we get

$$
\begin{gathered}
a_{40}=\frac{1}{\Gamma(1-v)}+\frac{1}{\Gamma(2-v)}+\frac{1}{\Gamma(3-v)}, \quad a_{41}=0, \quad a_{42}=0, \\
a_{43}=-\frac{1}{\Gamma(2-v)}-\frac{2}{\Gamma(3-v)}, \quad a_{44}=0, \quad a_{45}=\frac{1}{\Gamma(3-v)} .
\end{gathered}
$$

From the mask FDD- $i(i=1,2,3,4)$, we take three direction angles $\alpha=0, \pi / 4, \pi / 2$ in every quadrant, respectively, thus the $v$-order FDD at any point along twelve directions can implement in $[0,2 \pi]$ in an image $S(x, y)$, which can enhance its anti-rotation capability. But note that the coefficients (3.8), (3.10), (3.15), and (3.24) are same as (3.29), (3.17), (3.22), and (3.31) respectively. Therefore, the mask FDD- $i(i=1,2,3,4)$ can actually achieve FDD along the eight directions in four quadrants, where the coefficients of the masks are (3.8), (3.9), (3.16), (3.17), (3.22), (3.23), (3.30), and (3.31), respectively. These fractional differential coefficients of the eight directions are different. This is the biggest difference between FDD and general fractional differentiation. Generally, FDD-1, FDD-2, FDD-3, and FDD-4 are collectively called FDD operator.

Digital image processing is based on direct processing discrete pixels, while the numerical calculation of FDD is to implement fractional space filter by FDD operator 
convolution. The principle of space filter is to move the operator point by point. In digital image $S(x, y)$, FDD operator is used to filter with the convolution algorithms of FDD-1, FDD2, FDD-3, and FDD-4, respectively

$$
\begin{aligned}
& S_{1}^{(v)}(x, y)=\sum_{i=0}^{2} \sum_{j=0}^{2}(\mathrm{FDD}-1)(i, j) S(x+i, y+j), \\
& S_{2}^{(v)}(x, y)=\sum_{i=0}^{2} \sum_{j=-2}^{0}(\mathrm{FDD}-2)(i, j) S(x+i, y+j), \\
& S_{3}^{(v)}(x, y)=\sum_{i=-2}^{0} \sum_{j=-2}^{0}(\mathrm{FDD}-3)(i, j) S(x+i, y+j), \\
& S_{4}^{(v)}(x, y)=\sum_{i=-2}^{0} \sum_{j=0}^{2}(\mathrm{FDD}-4)(i, j) S(x+i, y+j) .
\end{aligned}
$$

Since the coefficients of FDD along the eight directions are different and it can effectively enlarge the differences among image textures, FDD can enhance not only the contour features in the smooth area nonlinearly, but the high-frequency edge feature in those areas where gray changes remarkably as well. To simplify the operation, according to the outputs comparison of the eight directions in four quadrants, the biggest one is selected as FDD grayscale. FDD algorithm for digital color image is similar to that for gray image, while the difference lies in that R, G, B components should do FDD, respectively, as the algorithm for gray image before composing the RGB color image. In other words, the calculation of mask convolution for color image is three times of that for gray image. there are strong interrelation among the RGB components, and the gray range is [0,255]. Thus, when the order $v \in[-1,1]$ is big or small, the interrelations can be broken especially in the case of nonlinearly enhancing the components, and the enhancement image may be distorted. For digital color image, it is better to take the format of $\mathrm{YCrCb}$ and HSV.

\section{Experiments and Results Analysis}

This section will demonstrate that FDD operator has better capability of texture enhancing for texture rich image. FDD is required in advance for an image by FDD-1, FDD-2, FDD3 , and FDD-4 masks, respectively, along eight directions. In four quadrants, the direction angles and coefficients of FDD operator are taken and found according to (3.8), (3.9), (3.16), (3.17), (3.22), (3.23), (3.30), and (3.31), respectively. And we can see that the differential coefficients along these eight directions in the image plane are not the same, which can reflect different fractional change rates along different directions and is benefiting to enlarge the differences among the image textures. The calculation rules of every mask FDD- $i$ refer to (3.32)-(3.35). Finally, we select the maximum output value of the eight directions in four quadrants as grayscale of the point. Note that FDD has a greater calculation range $[-1,1]$ than the transition width of general fractional differential order $(0,1)$. Clearly, when $-1 \leq v<0$, FDD is equivalent to the fractional directional integral operator. Some experimental results are shown in Figure 5. 


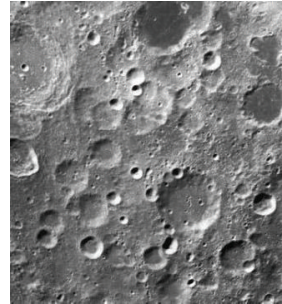

(a)

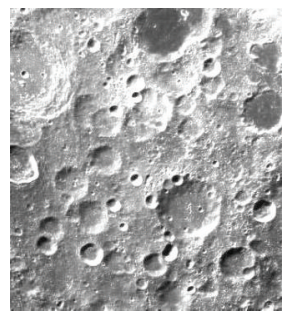

(e)

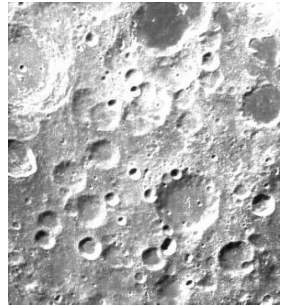

(b)

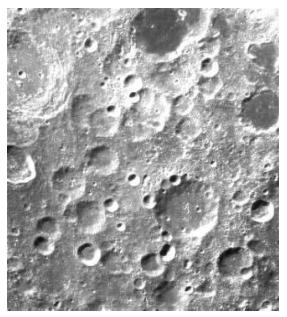

(f)

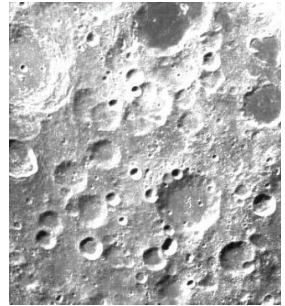

(c)

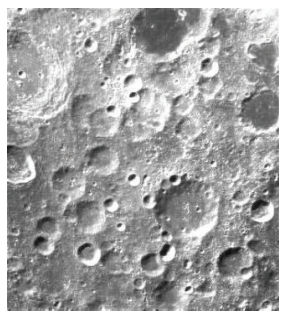

(g)

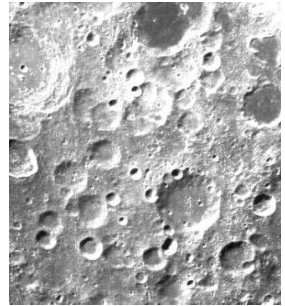

(d)

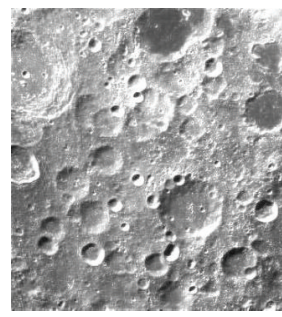

(h)

Figure 5: Texture information enhancement by FDD operator. (a) original image, (b) $v=-0.2$, (c) $v=-0.1$, (d) $v=0.1$, (e) $v=0.3$, (f) $v=0.5$, (g) $v=0.6$, and (h) $v=0.7$.

For quantity analysis, we calculate gray level cooccurrence matrix to describe the comprehensive information of the texture details like direction, neighboring distance, changing range, and the background. We pick up from gray level cooccurrence matrix five classical parameters of angle matrix, contrast, correlation, energy and homogeneity. The corresponding testing values of each parameter in the four angles can be obtained by taking $0^{\circ}, 45^{\circ}, 90^{\circ}$, and $135^{\circ}$, respectively. So, there are 16 testing values of parameters of gray level cooccurrence matrix. For the lunar surface in Figure 5, the parameters values of gray level cooccurrence matrix in 1-pixel distance are shown in Tables 1 and 2.

The visual analysis of the above data and discussion of the relationship between order $v$ and texture-enhancing details in the directions of $0^{\circ}, 45^{\circ}, 90^{\circ}$, and $135^{\circ}$ are integrated as nonlinear curves by four parameters: contrast, correlation, energy, and homogeneity, shown in Figure 6.

Tables 1 and 2 and Figure 6 show that, no matter what the angle is, the change of texture-enhancement has some relations with the order $v$. From (a) in Figure 6, we see that four contrast curves of enhancement image are above that of the original image. Image contrast can be interpreted as image clarity, that is, the clarity of image texture. The deeper the texture channels become, the greater the contrast is, as a result, the image looks clearer. The (b) in Figure 6 shows that the correlation of gray level cooccurrence matrix is always below that of the original image and becomes weaker with the increasing of $v$ when $-1 \leq v \leq 1$, that is to say, when the correlation decreases, the texture details become clearer. From (c) in Figure 6, we observe that the energy curve first decreases and then increases during $-1 \leq v \leq 1$. Particularly, when $v \in[-1,-0.5]$, the energy decreases and is above that of the original image; when $v \in(-0.5,0.5]$, the energy continues to decrease and is less than that of the original image; when $v \in[0.5,1]$, the energy increases with the increasing of $v$ and is still below that of the original image. Since the energy is the sum of square of gray level cooccurrence matrix element and reflects the uniformity of image gray level distribution 
Table 1: Gray level cooccurrence matrix in $0^{\circ}$ and $45^{\circ}$ directions.

\begin{tabular}{lcccccccc}
\hline $\begin{array}{l}\text { GLCM } \\
\text { order }\end{array}$ & \multicolumn{7}{c}{$0^{\circ}$} & \multicolumn{7}{c}{4} & \multicolumn{5}{c}{$45^{\circ}$} \\
\hline Original & 1.5065 & 0.8804 & 0.0334 & 0.6722 & 1.8104 & 0.8564 & 0.0296 & 0.6405 \\
-1.0 & 2.5271 & 0.8677 & 0.0493 & 0.6490 & 3.1893 & 0.8331 & 0.0449 & 0.6145 \\
-0.9 & 2.5316 & 0.8679 & 0.0462 & 0.6464 & 3.2058 & 0.8327 & 0.0419 & 0.6113 \\
-0.8 & 2.5437 & 0.8677 & 0.0430 & 0.6434 & 3.2350 & 0.8318 & 0.0389 & 0.6077 \\
-0.7 & 2.5595 & 0.8674 & 0.0400 & 0.6402 & 3.2609 & 0.8311 & 0.0360 & 0.6044 \\
-0.6 & 2.5777 & 0.8667 & 0.0370 & 0.6367 & 3.2866 & 0.8301 & 0.0332 & 0.6001 \\
-0.5 & 2.5996 & 0.8658 & 0.0343 & 0.6336 & 3.3257 & 0.8283 & 0.0306 & 0.5972 \\
-0.4 & 2.6249 & 0.8646 & 0.0318 & 0.6303 & 3.3585 & 0.8268 & 0.0283 & 0.5930 \\
-0.3 & 2.6435 & 0.8635 & 0.0294 & 0.6265 & 3.3724 & 0.8259 & 0.0262 & 11.5895 \\
-0.2 & 2.6568 & 0.8626 & 0.0275 & 0.6231 & 3.3845 & 0.8250 & 0.0244 & 0.586 .3 \\
-0.1 & 2.6742 & 0.8612 & 0.0258 & 0.6192 & 3.3938 & 0.8239 & 0.0229 & 0.5836 \\
0 & 2.6799 & 0.8601 & 0.0245 & 0.6172 & 3.3945 & 0.8228 & 0.0217 & 0.5817 \\
0.1 & 2.6893 & 0.8586 & 0.0235 & 0.6167 & 3.3918 & 0.8217 & 0.0207 & 0.5805 \\
0.2 & 2.6962 & 0.8569 & 0.0226 & 0.6150 & 3.3955 & 0.8198 & 0.0199 & 0.5787 \\
0.3 & 2.6966 & 0.8550 & 0.0219 & 0.6143 & 3.3830 & 0.8182 & 0.0192 & 0.5773 \\
0.4 & 2.7052 & 0.8522 & 0.0214 & 0.6120 & 3.3831 & 0.8152 & 0.0188 & 0.5762 \\
0.5 & 2.7125 & 0.8485 & 0.0211 & 0.6112 & 3.3922 & 0.8106 & 0.0184 & 0.5741 \\
0.6 & 2.6820 & 0.8444 & 0.0214 & 0.6116 & 3.3610 & 0.8050 & 0.0186 & 0.5736 \\
0.7 & 2.5951 & 0.8398 & 0.0222 & 0.6140 & 3.2601 & 0.7988 & 0.0192 & 0.5761 \\
0.8 & 2.5032 & 0.8351 & 0.0231 & 0.6167 & 3.1603 & 0.7918 & 0.0200 & 0.5783 \\
0.9 & 2.3749 & 0.8301 & 0.0246 & 0.6228 & 3.0257 & 0.7835 & 0.0212 & 0.5824 \\
1.0 & 2.2807 & 0.8250 & 0.0257 & 0.6260 & 2.9306 & 0.7751 & 0.0221 & 0.5852 \\
\hline & & & & & & & & \\
\hline
\end{tabular}

and the texture coarseness, which means that the more concentrated on the distribution the gray level cooccurrence matrix elements are, the greater the energy is. On the contrary, when the energy is smaller, the texture changing becomes more inhomogeneous and irregular, and texture details are clearer. So, from the energy, texture details by FDD operator are better when $v \in(-0.5,1]$ in general. The Figure $6(\mathrm{~d})$ shows that the homogeneity of gray level coccurrence matrix is almost always below that of the original image and the trend is down then up. When $v \in[-1,0.5]$, the homogeneity decreases with the increasing of $v$, which means that the texture in different sections changes more dramatic and the texture details are clearer. When $v \in[0.5,1]$, the homogeneity correspondingly increases, that is, it changes little in different sections when $v \in[0.5,1]$. In summary, once one takes all the four parameters of gray-level cooccurrence matrix into account, the strongest points of FDD for texture-enhancing should focus on the range of $v \in(-0.5,1)$ in general. Here note that it is different from general fractional differential when $v=0$, the result by FDD operator does not denote the original image, but the image of series expression of directional derivative to original image by (2.11), and it does not do fractional differentiation with respect to direction variable $h$.

To further observe the texture-enhancing capability of FDD, we did a set of comparisons with the two algorithms, the general fractional differentiation, and the nonlinearly enhancing high-frequency components by Butterworth filter, for different kinds of textures. 
Table 2: Gray level cooccurrence matrix in $90^{\circ}$ and $135^{\circ}$ directions.

\begin{tabular}{|c|c|c|c|c|c|c|c|c|}
\hline \multirow{2}{*}{$\begin{array}{l}\text { GLCM } \\
\text { order }\end{array}$} & \multicolumn{4}{|c|}{$90^{\circ}$} & \multicolumn{4}{|c|}{$135^{\circ}$} \\
\hline & Contrast & Correlation & Energy & Homogeneity & Contrast & Correlation & Energy & Homogeneity \\
\hline Original & 1.5431 & 0.8775 & 0.0329 & 0.6698 & 3.0958 & 0.7544 & 0.0251 & 0.5918 \\
\hline-1.0 & 2.6409 & 0.8618 & 0.0489 & 0.6466 & 4.7323 & 0.7523 & 0.0386 & 0.5777 \\
\hline-0.9 & 2.6503 & 0.8617 & 0.0457 & 0.6434 & 4.7236 & 0.7535 & 0.0360 & 0.5755 \\
\hline-0.8 & 2.6629 & 0.8616 & 0.0425 & 0.6397 & 4.7278 & 0.7542 & 0.0334 & 0.5722 \\
\hline-0.7 & 2.6768 & 0.8613 & 0.0394 & 0.6365 & 4.7347 & 0.7547 & 0.0309 & 0.5691 \\
\hline-0.6 & 2.6884 & 0.8611 & 0.0366 & 0.6336 & 4.7340 & 0.7553 & 0.0285 & 0.5659 \\
\hline-0.5 & 2.7100 & 0.8601 & 0.0338 & 0.6299 & 4.7431 & 0.7552 & 0.0263 & 0.5628 \\
\hline-0.4 & 2.7361 & 0.8589 & 0.0314 & 0.6264 & 4.7569 & 0.7548 & 0.0244 & 0.5594 \\
\hline-0.3 & 2.7491 & 0.8581 & 0.0292 & 0.6234 & 4.7631 & 0.7541 & 0.0226 & 0.5564 \\
\hline-0.2 & 2.7569 & 0.8574 & 0.0274 & 0.6204 & 4.7642 & 0.7536 & 0.0211 & 0.5537 \\
\hline-0.1 & 2.7694 & 0.8563 & 0.0258 & 0.6180 & 4.7842 & 0.7517 & 0.0197 & 0.5505 \\
\hline 0 & 2.7745 & 0.8552 & 0.0244 & 0.6158 & 4.7784 & 0.7506 & 0.0187 & 0.5480 \\
\hline 0.1 & 2.7834 & 0.8537 & 0.0234 & 0.6139 & 4.7820 & 0.7486 & 0.0178 & 0.5448 \\
\hline 0.2 & 2.7848 & 0.8522 & 0.0225 & 0.6129 & 4.7626 & 0.7472 & 0.0171 & 0.5418 \\
\hline 0.3 & 2.7821 & 0.8505 & 0.0218 & 0.6117 & 4.7337 & 0.7456 & 0.0165 & 0.5400 \\
\hline 0.4 & 2.7845 & 0.8479 & 0.0213 & 0.6113 & 4.6981 & 0.7434 & 0.0162 & 0.5378 \\
\hline 0.5 & 2.7918 & 0.8441 & 0.0210 & 0.6089 & 4.6405 & 0.7409 & 0.0161 & 0.5362 \\
\hline 0.6 & 2.7606 & 0.8398 & 0.0212 & 0.6088 & 4.5055 & 0.7386 & 0.0164 & 0.5382 \\
\hline 0.7 & 2.6631 & 0.8356 & 0.0221 & 0.6126 & 4.2504 & 0.7376 & 0.0172 & 0.5434 \\
\hline 0.8 & 2.5679 & 0.8308 & 0.0229 & 0.6151 & 3.9849 & 0.7375 & 0.0181 & 0.5496 \\
\hline 0.9 & 2.4407 & 0.8254 & 0.0244 & 0.6205 & 3.6609 & 0.7381 & 0.0196 & 0.5592 \\
\hline 1.0 & 2.3475 & 0.8199 & 0.0256 & 0.6252 & 3.3956 & 0.7395 & 0.0208 & 0.5663 \\
\hline
\end{tabular}

The results are shown in Figure 7. In addition, for easy comparison of its enhancing results, the results of experiments are the iteration of corresponding pixels of original image and FDD image.

From Figure 7, we could find that the nonlinearly enhancing the high-frequency components by Butterworth filter suffers from one serious drawback when large structures are strongly enhanced. By contrast, the FDD allows the smooth enhancement of large structures. For the enhancement of small texture details, the FDD may have some advantages over Butterworth filter. And since the coefficients of general fractional differentiation are always the same along the eight directions in the image plane, which is not conducive to reflect the different change rates of the image along different directions. While the FDD coefficients along the eight directions in the image plane are not the same, which can reflect sufficiently different fractional change rates along different directions and is benefiting to enlarge the differences among the image textures. So the enhancement results by FDD are better than those by general fractional differential. FDD operator cannot only maintain the most energy of image on the low frequency, but also nonlinearly enhance its energy over intermediate and high frequencies, which leads to a richer texture details. For quantity analysis, we take information entropy [19] and average gradient [20,21] as parameters that are shown in Table 3. The entropy denotes a measure of the amount of image information. Certainly, texture information is also one of image information. If an image has no texture, the gray level 


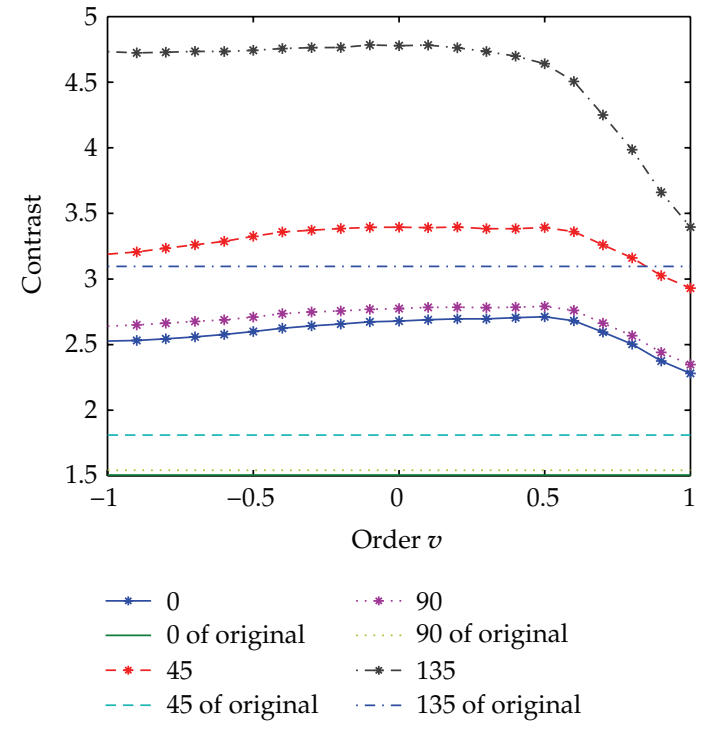

(a)

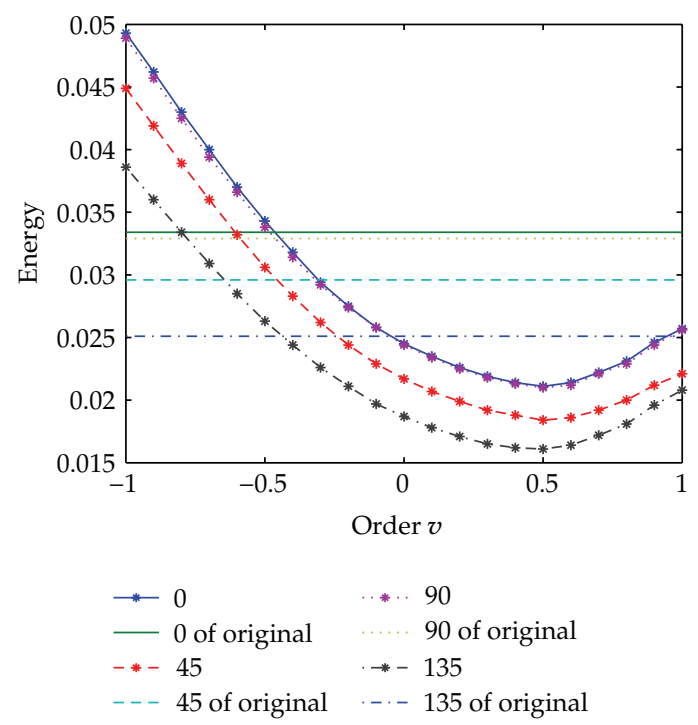

(c)

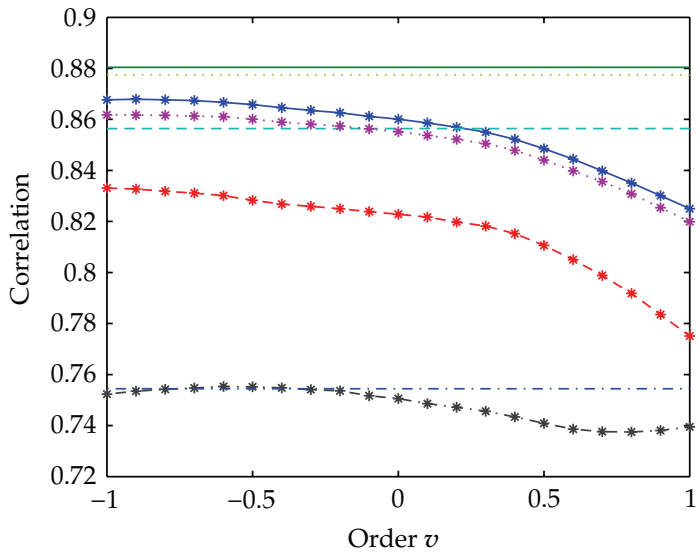

$$
\begin{aligned}
& \begin{array}{ll}
\rightarrow-0 & 0 \\
- & 0 \text { of original } \quad \cdots * \cdot 90 \\
\hline & \cdots \cdot-90 \text { of original }
\end{array} \\
& -*-45 \quad-*-135 \\
& \text {-- } 45 \text { of original _. - } 135 \text { of original }
\end{aligned}
$$

(b)

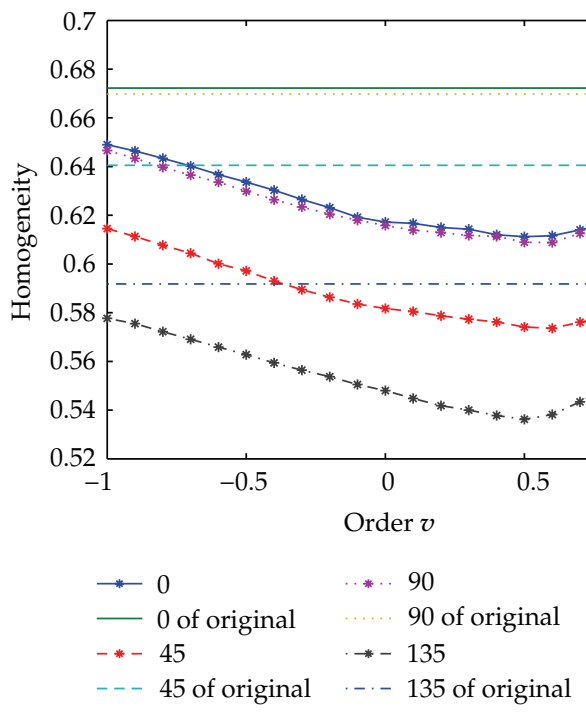

(d)

Figure 6: Curves of parameters of gray level cooccurrence matrix. (a) Contrast, (b) correlation, (c) energy, and (d) homogeneity.

cooccurrence matrix is almost a zero matrix and its entropy is close to zero. Otherwise, if an image has a great number of comprehensive texture details, its entropy is also greater. The average gradient can be sensitive to reflect the ability of contrast expression of small details, which can be used to evaluate image clarity. The experiment data of Figure 7 are shown in Table 3.

From Figure 7 and Table 3, we can see that, although the algorithm of the nonlinearly enhancing the high-frequency components by Butterworth filter can increase the average 


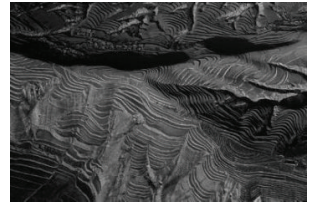

(a)

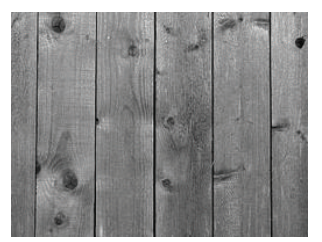

(e)

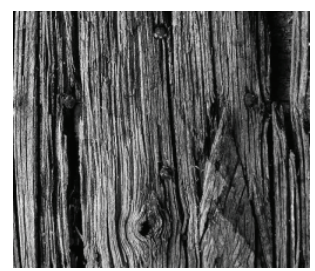

(i)

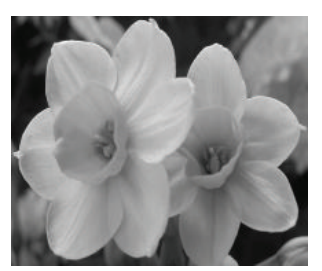

(m)

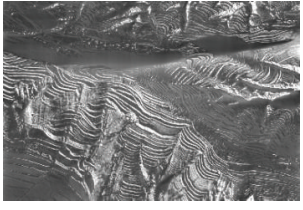

(b)

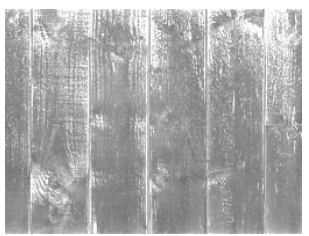

(f)

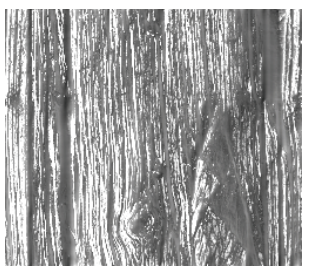

(j)

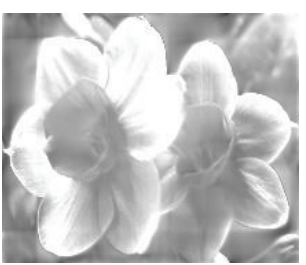

(n)

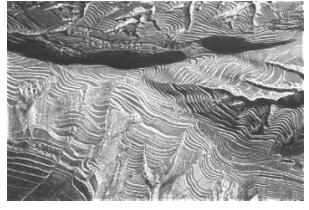

(c)

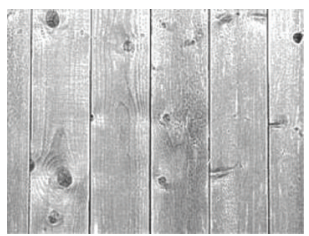

(g)

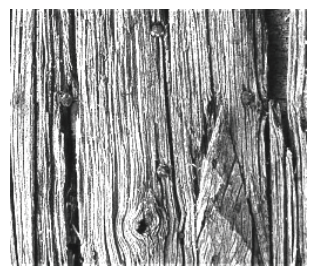

(k)

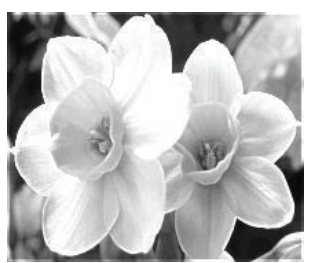

(o)

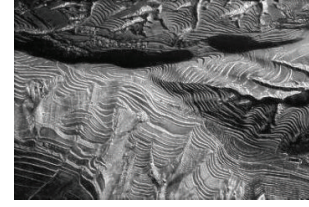

(d)

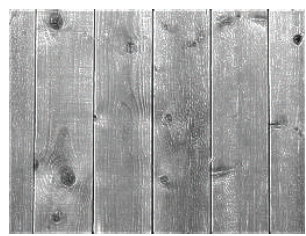

(h)

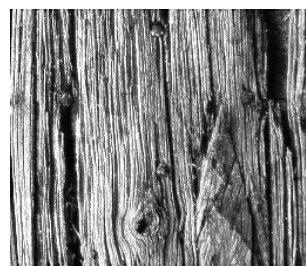

(1)

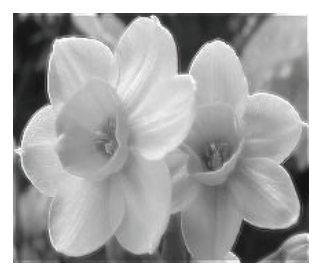

(p)

Figure 7: Texture-enhancement capability comparison. (a) original image, (b) the nonlinearly enhancing the high-frequency components by Butterworth filter, (c) general fractional differentiation of 0.6 order, (d) enhanced image by FDD operator of 0.8 order, (e) original image, (f) the nonlinearly enhancing the high-frequency components by Butterworth filter, (g) general fractional differentiation of 0.8 order, (h) enhanced image by FDD operator of 0.8 order, (i) original image, (j) the nonlinearly enhancing the highfrequency components by Butterworth filter, $(\mathrm{k})$ general fractional differentiation of 0.7 order, (l) enhanced image by FDD operator of 0.3 order, $(\mathrm{m})$ original image, $(\mathrm{n})$ the nonlinearly enhancing the high-frequency components by Butterworth filter, (o) general fractional differentiation of 0.6 order, and ( $p$ ) enhanced image by FDD operator of 0.9 order.

gradient, it only enhances the margin and losses a lot of texture details, thus it only has the minimum information entropy. Comparing with general fractional differential, the average gradient by FDD is slightly lower than it, however, the enhanced images by FDD have the biggest information entropy, which means FDD can enhance both the margins and the textures. Therefore, FDD could nonlinearly preserve the low-frequency contour feature in the smooth area to the furthest degree, nonlinearly enhance high-frequency marginal information in those areas where gray scale changes frequently, and nonlinearly enhance texture details in those areas where gray scale does not change evidently. Thus FDD could nonlinearly enhance the comprehensive texture details. 
Table 3: Information entropy and average gradient in Figure 7.

\begin{tabular}{lcc}
\hline Quantity figure & Information entropy & Average gradient \\
\hline (a) & 7.1169 & 12.7524 \\
(b) & 7.0527 & 18.5745 \\
(c) & 7.5542 & 24.2284 \\
(d) & 7.7479 & 21.3413 \\
(e) & 6.7022 & 8.1590 \\
(f) & 6.5945 & 9.9940 \\
(g) & 6.9191 & 14.3697 \\
(h) & 7.0042 & 12.3686 \\
(i) & 7.0699 & 20.1335 \\
(j) & 6.7949 & 21.9897 \\
(k) & 7.2170 & 31.1642 \\
(l) & 7.7158 & 28.0974 \\
(m) & 7.0124 & 4.7013 \\
(n) & 6.5731 & 4.9414 \\
(o) & 7.1646 & 7.1624 \\
(P) & 7.4953 & 5.5328 \\
\hline
\end{tabular}

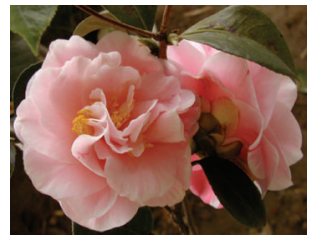

(a)

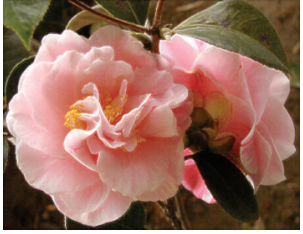

(b)

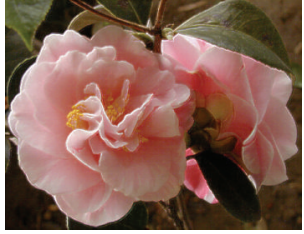

(c)

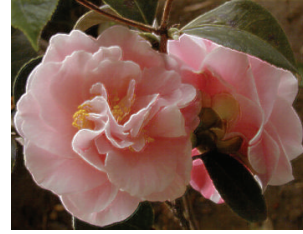

(d)

Figure 8: Color texture information enhanced by FDD operator. (a) original image, (b) $v=-0.1$, (c) $v=0.1$, (d) $v=0.2$.

For an RGB color image, the algorithm is similar to that for gray image. We do FDD for R, G, B component, respectively, then compose the RGB color image. The results are shown in Figure 8.

Observing carefully Figure 8, we see that the texture channels become deeper and the texture details are clearer by FDD operator. FDD operator not only maintains the most energy of image on the low frequency, but also nonlinearly enhance its energy over intermediate and high frequencies, which leads to a richer texture details. For the purpose of directly observing the change of grayscale, without losing generality, we take vertical projection ( $x$-coordinate) of gray level of (a) and (d) in Figure 8 as examples, to compare the change of gray-level values. The results are shown in Figure 9.

From Figure 9, we know that, the same as general fractional differential, FDD operator also enhances the high-frequency edge information with obvious enhancement effect. Moreover, comparing to integer-order differentiation, FDD keeps the envelope of gray level histogram distribution to some degree, and reserves the low-frequency contour information of the original image. After FDD to the original image, the result shows that the change magnitude of each point in the original image along eight directions in the four 


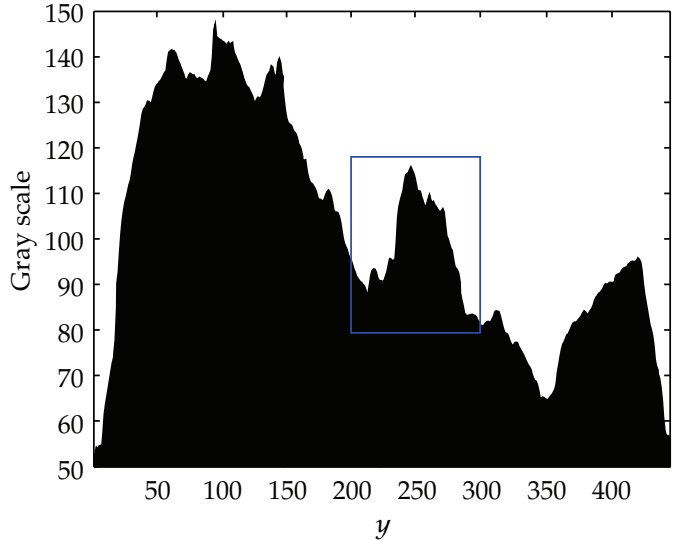

(a)

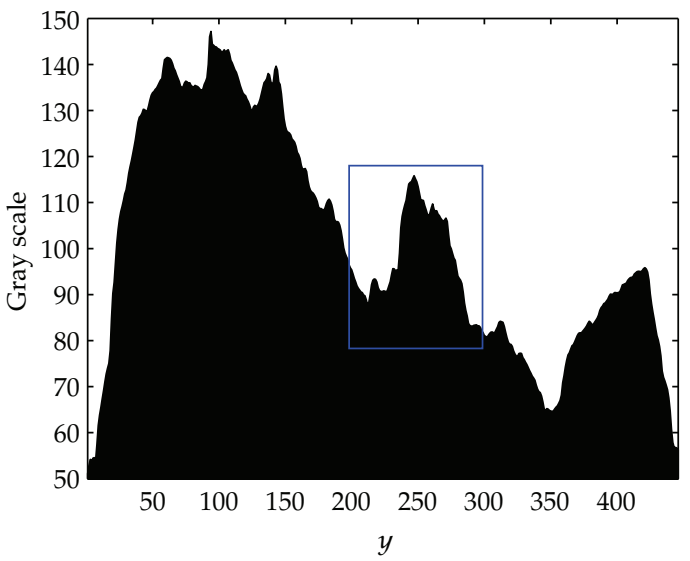

(c)

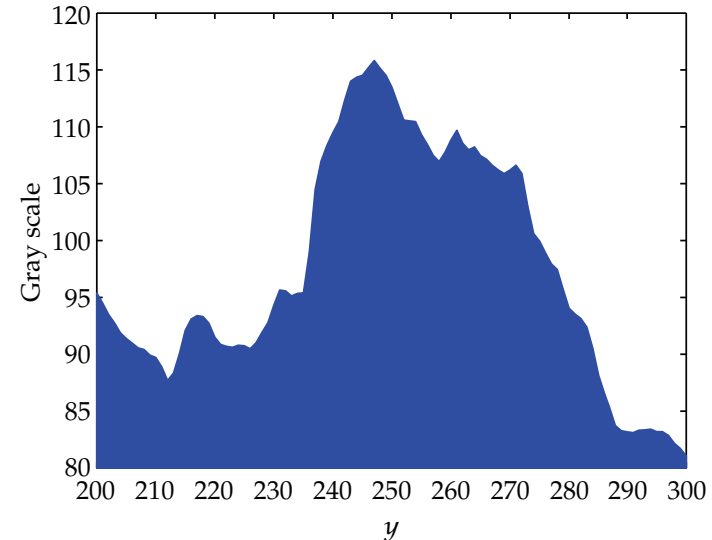

(b)

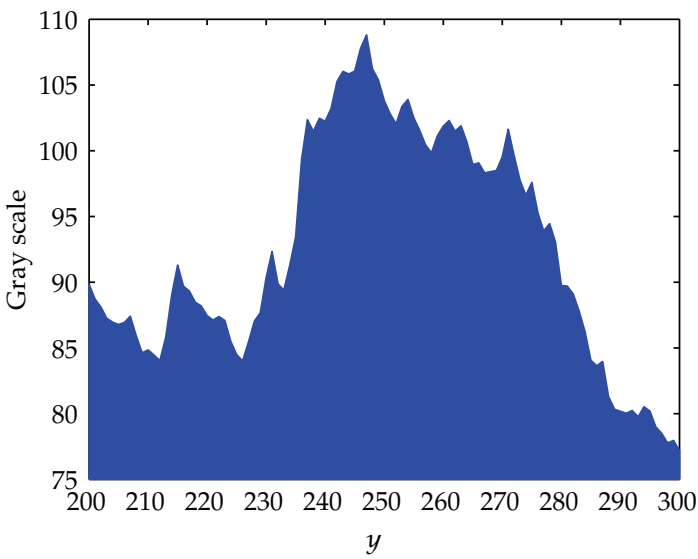

(d)

Figure 9: The vertical projections of gray scale of (a) and (d) in Figure 8. (a) the vertical projection of gray level of original image (a) in Figure 8, (b) part of (a), (c) the vertical projection of gray level of original image (d) in Figure 8, (d) part of (c).

quadrants. Therefore, FDD adds some burrs in the envelope curve of gray-level projection of the original image, which just denotes that the texture details are richer than the original image in these locations where there are many burrs and FDD can nonlinearly enhance texture details in those areas that gray levels have little changed, but integer-order differential is near to zero in those areas. In short, FDD not only nonlinearly preserves the contour feature in the smooth area, but also keeps high-frequency edge feature in those areas where gray changes remarkably and high-frequency texture detail feature in those areas where gray does not change evidently.

\section{Conclusion}

Fractional differentiation has played a very important role in digital image processing fields and more and more researchers begin to study it. This paper intends to propose a new mathematic approach (FDD) and deduce a new operator (FDD operator) obtained though 
the generalization of the Taylor formula for one-variable function to two-variable function and deduced though the power series expression of two-variable function. The particularity of FDD operator is that a directional derivative order between -1 and 1 favours detection selectivity, which expands the transition width of fractional differential order $0<v<1$. Since the FDD coefficients along the eight directions in the image plane are not the same, which can reflect sufficiently different fractional change rates along different directions and is benefiting to enlarge the differences among the image textures. So the enhancement results by FDD are better than those by general fractional differential. Experiments show that the FDD operator has excellent textural details enhancing capabilities for rich-grained digital images. For quantity analysis, from the different quantitative criteria, gray-level cooccurrence matrix, vertical projection ( $x$-coordinate) of gray-level, information entropy and average gradient, all show that FDD can nonlinearly enhance comprehensive texture details. FDD is a new method and technology for image-texture enhancement.

\section{Acknowledgments}

This work is supported by the National Natural Science Foundation of China (no. 60773168), the project of Science and Technology Department of Sichuan Province (no. 2011JY0077), the Natural Science Foundation of Chengdu University of China (no. 2010XJZ28), and the project of Chengdu City Economic and Information Technology Commission (no. 201201014).

\section{References}

[1] D. Baleanu, K. Diethelm, E. Scalas, and J. J. Trujillo, Fractional Calculus, vol. 3 of Series on Complexity, Nonlinearity and Chaos, World Scientific, Hackensack, NJ, USA, 2012.

[2] A. Babakhani, D. Baleanu, and R. Khanbabaie, "Hopf bifurcation for a class of fractional differential equations with delay," Nonlinear Dynamics, vol. 69, no. 3, pp. 721-729, 2012.

[3] S. Bhalekar, V. Daftardar-gejji, D. Baleanu, and R. Magin, "Generalized fractional order bloch equation with extended delay," International Journal of Bifurcation and Chaos, vol. 22, no. 4, Article ID 1250071, 15 pages, 2012.

[4] K. B. Oldham and J. Spanier, The Fractional Calculus, Academic Press, New York, NY, USA, 1974.

[5] A. A. Kilbas, H. M. Srivastava, and J. J. Trujillo, Theory and Applications of Fractional Differential Equations, vol. 204 of North-Holland Mathematics Studies, Elsevier Science B.V., Amsterdam, The Netherlands, 2006.

[6] M. A. E. Herzallah and D. Baleanu, "Fractional Euler-Lagrange equations revisited," Nonlinear Dynamics, vol. 69, no. 3, pp. 977-982, 2012.

[7] D. Baleanu, "New applications of fractional variational principles," Reports on Mathematical Physics, vol. 61, no. 2, pp. 199-206, 2008.

[8] M.-P. Chen and H. M. Srivastava, "Fractional calculus operators and their applications involving power functions and summation of series," Applied Mathematics and Computation, vol. 81, no. 2-3, pp. 287-304, 1997.

[9] N. Engheta, “On fractional calculus and fractional multipoles in electromagnetism," IEEE Transactions on Antennas and Propagation, vol. 44, no. 4, pp. 554-566, 1996.

[10] J. Sabatier, O. P. Agrawal, and J. A. Tenreiro Machado, Advances in Fractional Calculus: Theoretical Developments and Applications in Physics and Engineering, Springer, Dordrecht, The Netherlands, 2007.

[11] P. Janney and G. Geers, “Texture classification using invariant features of local textures," IET Image Processing, vol. 4, no. 3, pp. 158-171, 2010.

[12] R. M. Haralick, K. Shanmugam, and I. Dinstein, "Textural features for image classification," IEEE Transactions on Systems, Man and Cybernetics, vol. 3, no. 6, pp. 610-621, 1973.

[13] C. B. Gao, J. L. Zhou, J. R. Hu, and F. N. Lang, “Edge detection of colour image based on quaternion fractional differential," IET Image Processing, vol. 5, no. 3, pp. 261-272, 2011.

[14] C. B. Gao and J. L. Zhou, "Image enhancement based on quaternion fractional-order directional differentiation," Acta Automatica Sinica, vol. 37, no. 2, pp. 150-159, 2011 (Chinese). 
[15] C. Gao, J. Zhou, X. Zheng, and F. Lang, "Image enhancement based on improved fractional differentiation," Journal of Computational Information Systems, vol. 7, no. 1, pp. 257-264, 2011.

[16] Y.-F. Pu, J.-L. Zhou, and X. Yuan, "Fractional differential mask: a fractional differential-based approach for multiscale texture enhancement," IEEE Transactions on Image Processing, vol. 19, no. 2, pp. 491-511, 2010.

[17] C. Gao, "On power series of functions in several variables," College Mathematics, no. 3, pp. 125-129, 2007 (Chinese).

[18] Y. T. Bai, Y. F. Luo, and Y.X. Hu :, "The fractional integration and differentiation and their series expansions," Journal of Wuhan Technical University of Surveying and Mapping, vol. 18, no. 1, pp. 68-75, 1993 (Chinese).

[19] C. E. Shannon, "A mathematical theory of communication," The Bell System Technical Journal, vol. 27, pp. 379-423, 1948.

[20] John S. Weszka and Azriel Rosenfeld, "Histogram modification for threshold selection," IEEE Trans Syst Man Cybern, vol. 9, no. 1, pp. 38-52, 1979.

[21] A. M. Groenewald, E. Barnard, and E. C. Botha, "Related approaches to gradient-based thresholding," Pattern Recognition Letters, vol. 14, no. 7, pp. 567-572, 1993. 


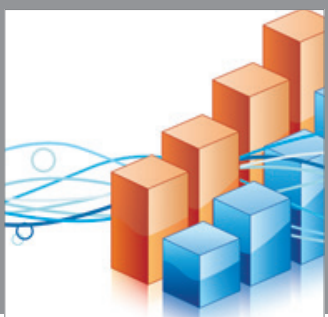

Advances in

Operations Research

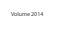

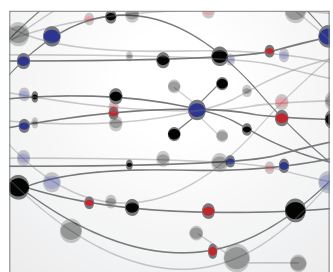

\section{The Scientific} World Journal
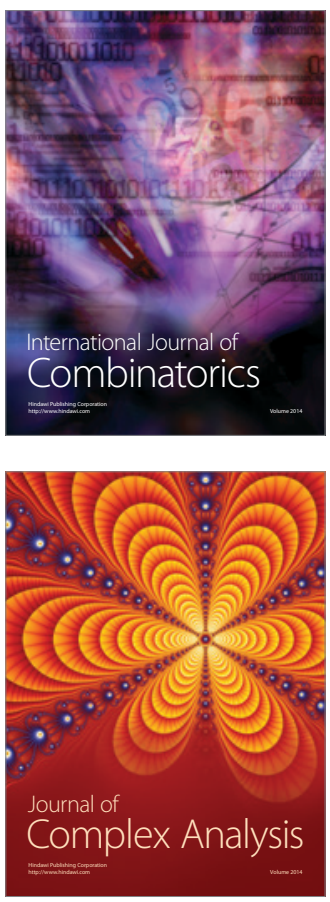

International Journal of

Mathematics and

Mathematical

Sciences
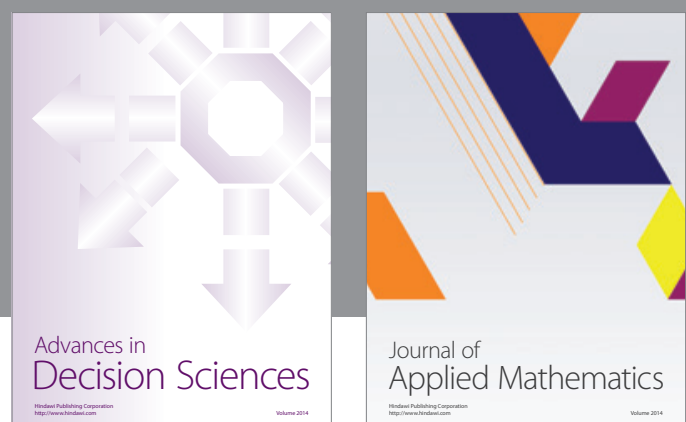

Journal of

Applied Mathematics
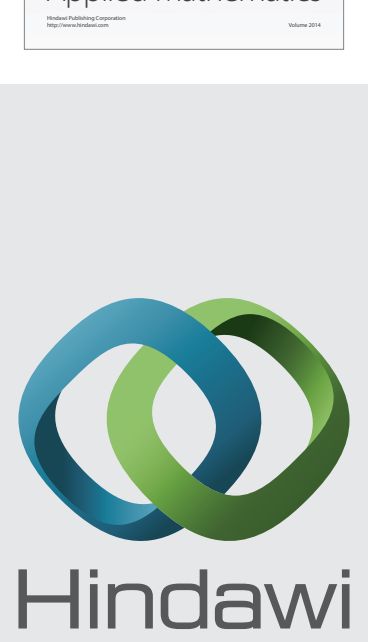

Submit your manuscripts at http://www.hindawi.com
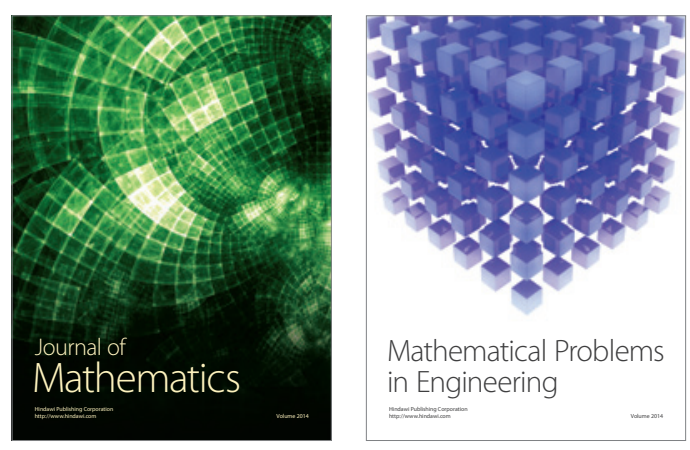

Mathematical Problems in Engineering
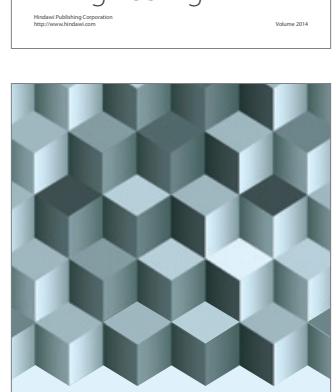

Journal of

Function Spaces
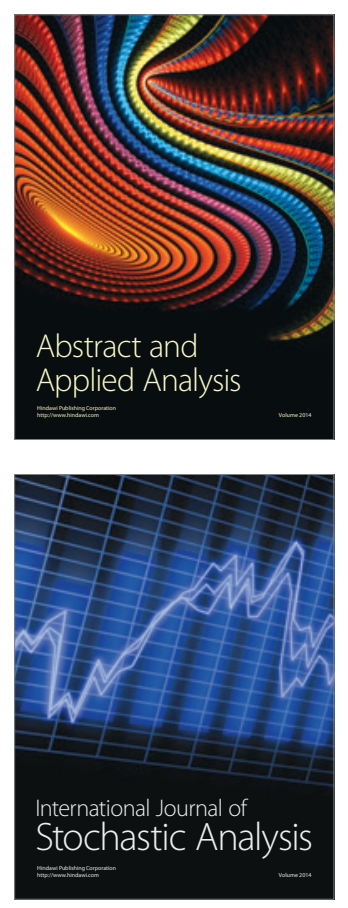

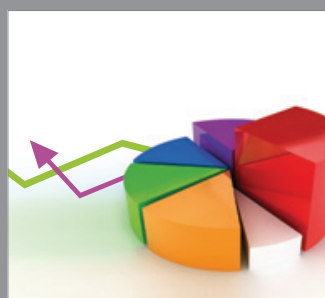

ournal of

Probability and Statistics

Promensencen
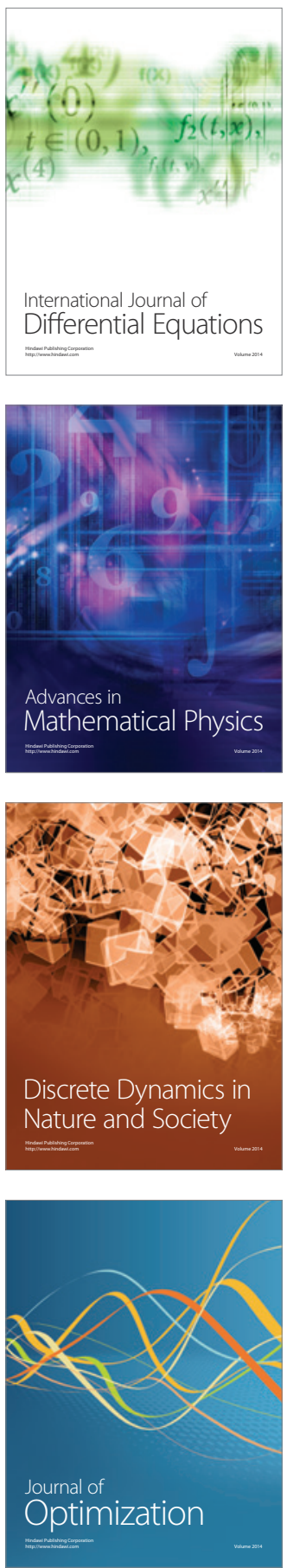